INTER NATIONAL MONETARY FUND
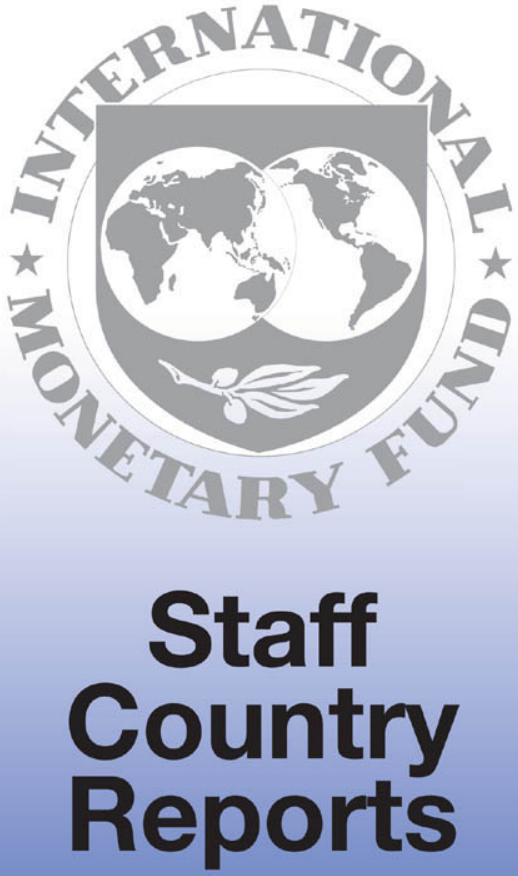


\title{
Brunei Darussalam: Statistical Appendix
}

This Statistical Appendix on Brunei Darussalam was prepared by a staff team of the International Monetary Fund as background documentation for the periodic consultation with the member country. It is based on the information available at the time it was completed on April 7, 2010. The views expressed in this document are those of the staff team and do not necessarily reflect the views of the government of Brunei Darussalam or the Executive Board of the IMF.

The policy of publication of staff reports and other documents by the IMF allows for the deletion of market-sensitive information.

\author{
Copies of this report are available to the public from \\ International Monetary Fund $\bullet$ Publication Services \\ $70019^{\text {th }}$ Street, N.W. • Washington, D.C. 20431 \\ Telephone: (202) 623-7430 • Telefax: (202) 623-7201 \\ E-mail: publications@imf.org Internet: http://www.imf.org
}

\section{International Monetary Fund Washington, D.C.}


This page intentionally left blank

CInternational Monetary Fund. Not for Redistribution 


\title{
INTERNATIONAL MONETARY FUND
}

\section{BRUNEI DARUSSALAM}

\section{Statistical Appendix}

\author{
Prepared by D. Filiz Unsal (APD)
}

Approved by Asia and Pacific Department

April 7, 2010

Contents

Page

1. Nominal GDP by Economic Activity, 2002-08

2. Quarterly Nominal GDP by Economic Activity, 2006-09 …………………..................

3. Nominal GDP by Expenditure, 2002-08 ………....................................................

4. Quarterly Nominal GDP by Expenditure, 2006-09.........................................................

5. Composition of Nominal GDP by Economic Activity, 2002-08 ….................................

6. Quarterly Composition of Nominal GDP by Economic Activity, 2006-09 ........................ $\underline{8}$

7. Composition of Nominal GDP by Expenditure, 2002-08 ……….................................

8. Quarterly Composition of Nominal GDP by Expenditure, 2006-09...............................10

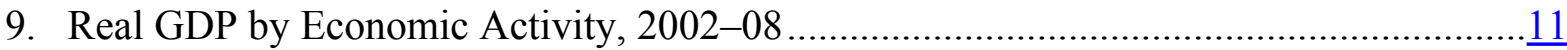

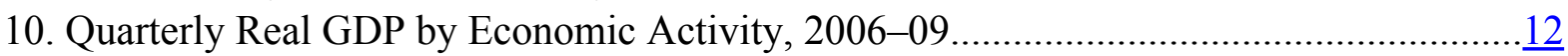

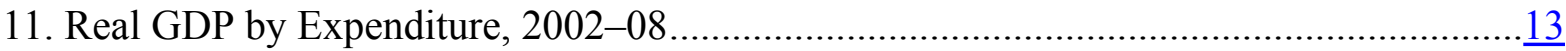

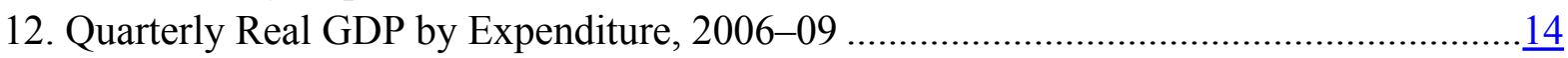

13. Growth in Real GDP by Economic Activity, 2003-08.................................................15

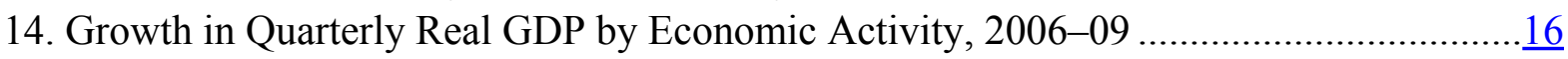

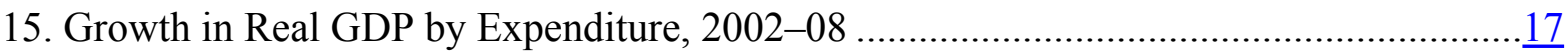

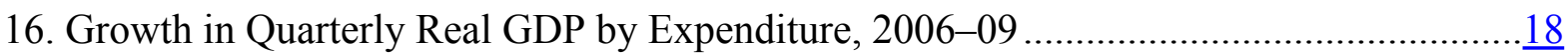

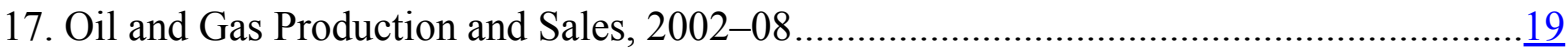

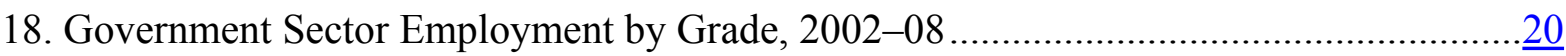

19. Private Sector Employment by Industry and Residency Status, 2008 ………………........

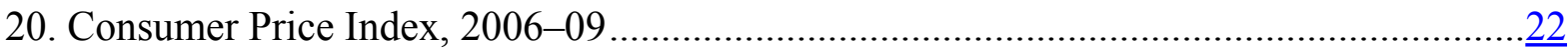

21. Composition of Government Revenue, 2002-2009....................................................

22. Composition of Quarterly Government Revenue, 2005/06-2009/10 ...............................

23. Composition of Government Expenditure and Budget Balance, 2002-2008/09................

24. Composition of Quarterly Government Expenditure and Budget Balance,

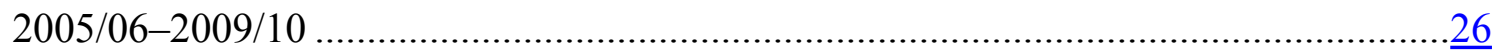

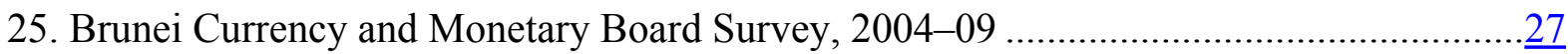

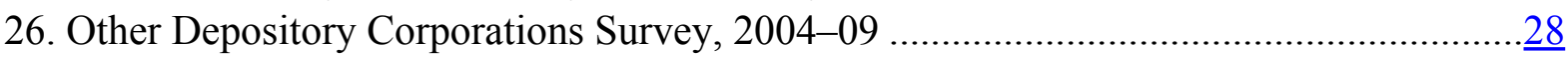

27. Depository Corporations Survey, 2004-09..................................................................... 
28. Direction of Loans by Commercial Banks, 2004-09.......................................................

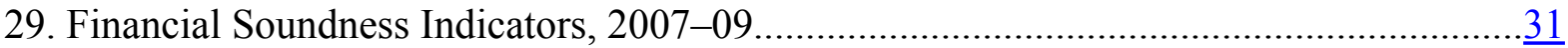

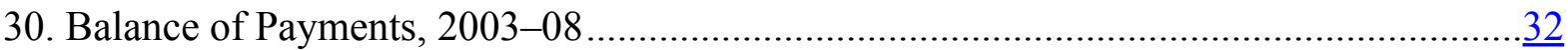

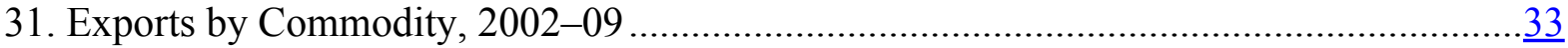

32. Value and Destination of Selected Exports, 2002-09 ………………….....................34

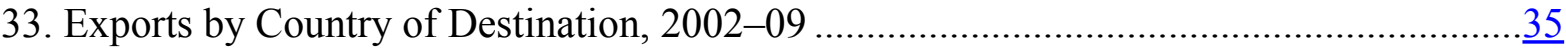

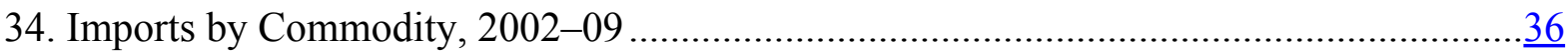

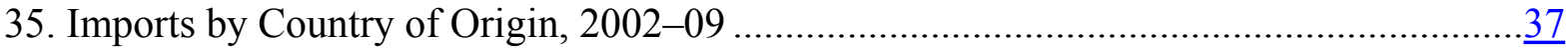

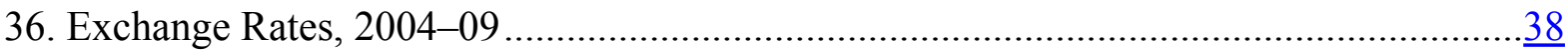


Table 1. Brunei Darussalam: Nominal GDP by Economic Activity, 2002-08

(In millions of Brunei dollars)

\begin{tabular}{|c|c|c|c|c|c|c|c|}
\hline & 2002 & 2003 & 2004 & 2005 & 2006 & 2007 & 2008 \\
\hline Gross domestic product (GDP) & $10,463.1$ & $11,424.2$ & $13,305.8$ & $15,864.1$ & $18,225.8$ & $18,458.4$ & $20,397.9$ \\
\hline Oil and gas sector & $5,531.6$ & $6,530.2$ & $8,236.4$ & $10,540.4$ & $12,491.0$ & $12,332.9$ & $14,300.0$ \\
\hline Oil and gas mining & $4,223.0$ & $5,079.5$ & $6,742.3$ & $8,868.0$ & $10,768.4$ & $10,603.7$ & $11,672.0$ \\
\hline Manufacture of liquefied natural gas (LNG) & $1,308.5$ & $1,450.7$ & $1,494.1$ & $1,672.4$ & $1,722.6$ & $1,729.3$ & $2,628.0$ \\
\hline Non-oil and gas sector & $4,931.5$ & $4,894.0$ & $5,069.3$ & $5,323.4$ & $5,734.7$ & $6,125.5$ & $6,097.9$ \\
\hline Government & $1,710.2$ & $1,688.9$ & $1,785.3$ & $1,847.0$ & $2,008.5$ & $2,239.4$ & $2,152.2$ \\
\hline Private sector & $3,221.2$ & $3,205.1$ & $3,284.0$ & $3,476.4$ & $3,726.2$ & $3,886.1$ & $3,945.7$ \\
\hline Non-oil and gas sector & $4,931.5$ & $4,894.0$ & $5,069.3$ & $5,323.4$ & $5,734.7$ & $6,125.5$ & $6,097.9$ \\
\hline Vegetables, fruits and other agriculture & 22.5 & 27.3 & 25.2 & 23.6 & 20.5 & 24.6 & 29.9 \\
\hline Livestock and poultry & 35.8 & 33.3 & 43.6 & 34.8 & 37.9 & 41.3 & 46.2 \\
\hline Forestry & 4.2 & 4.4 & 5.1 & 5.3 & 5.4 & 4.9 & 6.6 \\
\hline Fishery & 52.0 & 74.7 & 76.1 & 86.4 & 64.9 & 56.5 & 46.9 \\
\hline Manufacture of wearing apparel and textile & 247.9 & 211.6 & 181.4 & 197.3 & 124.0 & 116.4 & 103.2 \\
\hline Other manufacturing & 84.6 & 87.4 & 92.4 & 82.7 & 78.7 & 70.5 & 64.9 \\
\hline Electricity and water & 92.3 & 98.0 & 95.3 & 113.3 & 103.8 & 111.5 & 115.0 \\
\hline Construction & 390.7 & 401.1 & 422.8 & 418.5 & 539.5 & 530.4 & 534.4 \\
\hline Wholesale and retail trade & 403.4 & 414.5 & 443.6 & 457.8 & 486.3 & 549.0 & 558.0 \\
\hline Water transport & 109.1 & 135.1 & 134.2 & 143.3 & 172.3 & 149.5 & 145.5 \\
\hline Air transport & 118.8 & 110.3 & 116.2 & 115.7 & 114.9 & 116.6 & 114.3 \\
\hline Other transport services & 34.9 & 32.8 & 32.3 & 44.1 & 69.2 & 95.8 & 108.5 \\
\hline Communication & 162.6 & 166.7 & 171.0 & 153.4 & 147.2 & 149.5 & 165.2 \\
\hline Finance & 352.2 & 355.1 & 375.4 & 472.3 & 552.8 & 579.0 & 593.2 \\
\hline Real estate and ownership of dwellings & 406.4 & 395.0 & 413.8 & 412.1 & 415.4 & 427.2 & 433.3 \\
\hline Hotels and restaurants & 62.2 & 63.0 & 67.5 & 58.2 & 66.6 & 57.7 & 57.5 \\
\hline Private health and education services & 54.9 & 59.9 & 61.2 & 105.7 & 108.9 & 107.2 & 100.1 \\
\hline Business services & 477.6 & 432.7 & 437.1 & 462.0 & 524.3 & 599.2 & 619.8 \\
\hline Domestic services & 47.4 & 48.4 & 49.5 & 50.2 & 50.8 & 51.3 & 51.9 \\
\hline Other private services & 61.9 & 53.8 & 40.3 & 39.7 & 43.0 & 48.0 & 51.2 \\
\hline Government services & $1,710.2$ & $1,688.9$ & $1,785.3$ & $1,847.0$ & $2,008.5$ & $2,239.4$ & $2,152.2$ \\
\hline \multicolumn{8}{|l|}{ Memorandum items: } \\
\hline Population & 344,200 & 349,600 & 359,700 & 370,100 & 383,000 & 390,000 & 398,000 \\
\hline GDP per capita (in Brunei dollars) & $30,398.0$ & $32,678.0$ & $36,991.0$ & $42,864.0$ & $47,587.0$ & $47,329.0$ & $51,251.0$ \\
\hline
\end{tabular}

Source: Department of Economic Planning and Development (JPKE). 
Table 2. Brunei Darussalam: Quarterly Nominal GDP by Economic Activity, 2006-09

(In millions of Brunei dollars)

\begin{tabular}{|c|c|c|c|c|c|c|c|c|c|c|c|c|c|}
\hline & \multicolumn{4}{|c|}{2006} & \multicolumn{4}{|c|}{2007} & \multicolumn{4}{|c|}{2008} & \multirow{2}{*}{$\frac{2009}{\mathrm{Q} 1}$} \\
\hline & Q1 & Q2 & Q3 & $\overline{Q 4}$ & Q1 & Q2 & Q3 & $\overline{Q 4}$ & Q1 & Q2 & Q3 & $\overline{\text { Q4 }}$ & \\
\hline Gross domestic product (GDP) & $4,637.6$ & $4,478.6$ & $4,737.7$ & $4,372.0$ & $4,231.2$ & $4,278.3$ & $4,494.8$ & $5,454.2$ & $5,083.2$ & $5,070.5$ & $5,594.8$ & $4,649.3$ & $3,563.7$ \\
\hline Oil and gas sector & $3,132.8$ & $3,110.3$ & $3,322.6$ & $2,925.2$ & $2,652.7$ & $2,819.9$ & $3,017.9$ & $3,842.5$ & $3,062.0$ & $3,624.2$ & $4,068.8$ & $3,005.1$ & $2,066.2$ \\
\hline Oil and gas mining & $2,737.9$ & $2,702.9$ & $2,868.4$ & $2,459.2$ & $2,281.5$ & $2,419.6$ & $2,608.2$ & $3,294.4$ & $3,173.0$ & $3,154.3$ & $3,468.6$ & $1,876.0$ & $1,418.5$ \\
\hline Manufacture of liquefied natural gas (LNG) & 394.9 & 407.5 & 454.3 & 466.0 & 371.2 & 400.3 & 409.7 & 548.1 & 428.9 & 469.9 & 600.2 & $1,129.1$ & 647.6 \\
\hline Non-oil and gas sector & $1,504.8$ & $1,368.3$ & $1,414.9$ & $1,446.8$ & $1,578.5$ & $1,458.4$ & $1,476.9$ & $1,611.7$ & $1,481.2$ & $1,446.3$ & $1,526.1$ & $1,644.3$ & $1,497.5$ \\
\hline Government & 558.3 & 434.6 & 488.4 & 527.2 & 618.8 & 488.6 & 489.0 & 642.9 & 503.4 & 493.8 & 503.9 & 651.1 & 512.7 \\
\hline Private sector & 946.5 & 933.6 & 926.4 & 919.7 & 959.7 & 969.8 & 987.9 & 968.8 & 977.8 & 952.5 & $1,022.2$ & 993.2 & 984.8 \\
\hline Non-oil and gas sector & $1,504.8$ & $1,368.3$ & $1,414.9$ & $1,446.8$ & $1,578.5$ & $1,458.4$ & $1,476.9$ & $1,611.7$ & $1,481.2$ & $1,446.3$ & $1,526.1$ & $1,644.3$ & $1,497.5$ \\
\hline Vegetables, fruits \& other agriculture & 4.6 & 5.2 & 5.4 & 5.3 & 5.5 & 5.8 & 7.2 & 6.1 & 6.5 & 7.0 & 7.7 & 8.7 & 5.1 \\
\hline Livestock and poultry & 9.1 & 8.9 & 9.7 & 10.2 & 10.4 & 10.3 & 10.3 & 10.3 & 10.7 & 11.3 & 12.3 & 11.8 & 16.2 \\
\hline Forestry & 1.2 & 1.5 & 1.6 & 1.1 & 1.2 & 1.4 & 1.1 & 1.2 & 1.4 & 2.1 & 1.9 & 1.2 & 1.0 \\
\hline Fishery & 20.0 & 16.0 & 16.0 & 12.9 & 14.2 & 14.1 & 14.1 & 14.1 & 13.2 & 11.1 & 10.9 & 11.7 & 12.3 \\
\hline Manufacture of wearing apparel \& textile & 39.2 & 25.7 & 29.0 & 30.1 & 34.8 & 27.9 & 23.7 & 30.1 & 41.5 & 19.5 & 21.3 & 20.9 & 20.7 \\
\hline Other manufacturing & 19.0 & 23.5 & 19.5 & 16.7 & 19.6 & 22.2 & 15.4 & 13.3 & 17.7 & 20.0 & 16.5 & 10.7 & 19.7 \\
\hline Electricity and water & 22.8 & 23.3 & 29.0 & 28.7 & 27.9 & 24.4 & 29.8 & 29.4 & 28.1 & 26.6 & 30.0 & 30.3 & 29.3 \\
\hline Construction & 127.9 & 154.0 & 142.1 & 115.5 & 116.7 & 110.4 & 172.5 & 130.8 & 119.7 & 105.0 & 176.1 & 133.6 & 120.9 \\
\hline Wholesale and retail trade & 142.3 & 110.8 & 122.5 & 110.6 & 153.6 & 135.1 & 140.6 & 119.8 & 149.1 & 139.6 & 141.6 & 127.7 & 158.5 \\
\hline Water transport & 67.1 & 30.0 & 38.2 & 37.1 & 52.2 & 22.7 & 39.0 & 35.7 & 49.8 & 22.3 & 38.4 & 35.0 & 52.0 \\
\hline Air transport & 27.1 & 28.1 & 34.1 & 25.6 & 28.0 & 28.9 & 36.2 & 23.4 & 29.1 & 25.7 & 38.6 & 20.8 & 30.4 \\
\hline Other transport services & 34.4 & 13.3 & 9.1 & 12.4 & 39.6 & 30.5 & 13.5 & 12.2 & 44.8 & 36.7 & 14.8 & 12.2 & 44.7 \\
\hline Communication & 16.6 & 25.7 & 68.1 & 36.8 & 16.5 & 26.0 & 71.4 & 35.7 & 17.3 & 27.8 & 73.9 & 46.2 & 17.5 \\
\hline Finance & 110.5 & 122.6 & 142.1 & 177.5 & 113.2 & 126.6 & 151.5 & 187.7 & 116.1 & 127.3 & 159.9 & 190.0 & 119.5 \\
\hline Real estate \& ownership of dwellings & 103.9 & 104.4 & 103.4 & 103.7 & 106.0 & 107.0 & 107.2 & 107.0 & 107.6 & 108.7 & 108.7 & 108.3 & 107.5 \\
\hline Hotels and restaurants & 19.9 & 18.5 & 13.1 & 15.0 & 15.1 & 15.2 & 12.3 & 15.1 & 15.3 & 14.4 & 12.5 & 15.3 & 16.0 \\
\hline Private health and education services & 25.3 & 29.8 & 28.6 & 25.2 & 28.4 & 32.0 & 22.7 & 24.0 & 32.4 & 21.7 & 22.1 & 23.8 & 35.6 \\
\hline Business services & 132.5 & 166.4 & 91.4 & 133.9 & 153.2 & 200.1 & 94.9 & 150.9 & 156.2 & 196.8 & 106.4 & 160.4 & 157.3 \\
\hline Domestic services & 12.8 & 12.8 & 12.6 & 12.8 & 12.8 & 12.8 & 12.8 & 12.8 & 13.0 & 13.0 & 13.0 & 13.0 & 13.0 \\
\hline Other private services & 10.3 & 13.1 & 11.0 & 8.6 & 10.5 & 16.5 & 11.7 & 9.3 & 8.1 & 16.0 & 15.6 & 11.5 & 7.7 \\
\hline Government Services & 558.3 & 434.6 & 488.4 & 527.2 & 618.8 & 488.6 & 489.0 & 642.9 & 503.4 & 493.8 & 503.9 & 651.1 & 512.7 \\
\hline
\end{tabular}

Source: Department of Economic Planning and Development (JPKE).

(CInternational Monetary Fund. Not for Redistribution 
Table 3. Brunei Darussalam: Nominal GDP by Expenditure, 2002-08

(In millions of Brunei dollars)

\begin{tabular}{|c|c|c|c|c|c|c|c|}
\hline & 2002 & 2003 & 2004 & 2005 & 2006 & 2007 & 2008 \\
\hline Gross domestic product (GDP) & $10,463.1$ & $11,424.2$ & $13,305.8$ & $15,864.1$ & $18,225.8$ & $18,458.4$ & $20,397.9$ \\
\hline Domestic demand & $7,745.9$ & $7,590.5$ & $8,263.2$ & $8,286.8$ & $8,804.7$ & $10,294.2$ & $9,892.4$ \\
\hline Consumption & $5,521.5$ & $5,870.6$ & $6,468.7$ & $6,483.6$ & $6,902.3$ & $7,897.4$ & $7,104.4$ \\
\hline Personal consumption expenditure & $2,678.7$ & $3,121.0$ & $3,534.4$ & $3,563.2$ & $3,610.4$ & $3,722.0$ & $3,608.0$ \\
\hline Government consumption expenditure & $2,842.8$ & $2,749.5$ & $2,934.3$ & $2,920.4$ & $3,291.9$ & $4,175.4$ & $3,496.4$ \\
\hline Gross capital formation & $2,224.4$ & $1,720.0$ & $1,794.5$ & $1,803.2$ & $1,902.4$ & $2,396.8$ & $2,788.0$ \\
\hline Gross fixed investment & $2,217.7$ & $1,711.8$ & $1,787.2$ & $1,801.3$ & $1,907.4$ & $2,398.5$ & $2,788.9$ \\
\hline Construction & $1,151.4$ & $1,098.8$ & $1,158.3$ & $1,206.9$ & $1,221.5$ & $1,383.3$ & $1,601.0$ \\
\hline Machinery and equipment & $1,066.4$ & 613.0 & 628.9 & 594.3 & 685.9 & $1,015.2$ & $1,187.9$ \\
\hline Change in stocks & 6.7 & 8.2 & 7.3 & 1.9 & -5.1 & -1.7 & -0.9 \\
\hline Net exports of goods and services & $2,667.6$ & $3,806.5$ & $4,755.2$ & $6,802.4$ & $8,476.5$ & $7,375.1$ & $10,338.6$ \\
\hline Exports of goods and services & $7,023.0$ & $7,915.7$ & $9,154.3$ & $11,131.6$ & $13,072.4$ & $12,524.6$ & $15,971.4$ \\
\hline Imports of goods and services & $4,355.4$ & $4,109.2$ & $4,399.1$ & $4,329.2$ & $4,595.9$ & $5,149.5$ & $5,632.8$ \\
\hline Statistical discrepancy (residual to tally up) & 49.6 & 27.1 & 287.5 & 774.8 & 944.7 & 789.2 & 166.8 \\
\hline
\end{tabular}

Source: Department of Economic Planning and Development (JPKE). 
Table 4. Brunei Darussalam: Quarterly Nominal GDP by Expenditure, 2006-09

(In millions of Brunei dollars)

\begin{tabular}{|c|c|c|c|c|c|c|c|c|c|c|c|c|c|}
\hline & \multicolumn{4}{|c|}{2006} & \multicolumn{4}{|c|}{2007} & \multicolumn{4}{|c|}{2008} & \multirow{2}{*}{$\frac{2009}{\mathrm{Q} 1}$} \\
\hline & Q1 & Q2 & Q3 & $\overline{\text { Q4 }}$ & Q1 & Q2 & Q3 & $\overline{\mathrm{Q} 4}$ & Q1 & Q2 & Q3 & $\overline{\text { Q4 }}$ & \\
\hline Gross domestic product (GDP) & $4,637.6$ & $4,478.6$ & $4,737.7$ & $4,372.0$ & $4,231.2$ & $4,278.3$ & $4,494.8$ & $5,454.2$ & $5,083.2$ & $5,070.5$ & $5,594.8$ & $4,649.3$ & $3,563.7$ \\
\hline Domestic demand & $2,234.1$ & $2,186.6$ & $2,213.0$ & $2,171.0$ & $2,434.0$ & $2,360.5$ & $2,830.5$ & $2,669.1$ & $2,407.6$ & $2,292.7$ & $2,645.8$ & $2,546.4$ & $2,635.5$ \\
\hline Consumption & $1,797.3$ & $1,716.6$ & $1,739.8$ & $1,648.6$ & $1,898.2$ & $1,882.9$ & $2,105.1$ & $2,011.2$ & $1,774.4$ & $1,747.1$ & $1,799.3$ & $1,783.6$ & $1,862.2$ \\
\hline Personal consumption expenditure & 867.9 & 959.9 & 967.6 & 815.1 & 883.9 & 982.0 & 998.6 & 857.5 & 867.6 & 913.6 & 944.2 & 882.6 & 909.5 \\
\hline Government consumption expenditure & 929.4 & 756.7 & 772.2 & 833.5 & $1,014.3$ & 900.9 & $1,106.5$ & $1,153.7$ & 906.8 & 833.5 & 855.1 & 901.0 & 952.7 \\
\hline Gross capital formation & 436.8 & 470.0 & 473.2 & 522.4 & 535.8 & 477.6 & 725.5 & 658.0 & 633.2 & 545.6 & 846.5 & 762.8 & 773.3 \\
\hline Gross fixed investment & 437.2 & 472.4 & 475.0 & 522.8 & 534.3 & 477.7 & 726.4 & 660.0 & 635.1 & 544.6 & 847.3 & 762.0 & 772.7 \\
\hline Construction & 291.3 & 296.1 & 317.6 & 316.5 & 285.8 & 264.9 & 388.9 & 443.7 & 416.3 & 296.1 & 425.4 & 463.2 & 424.2 \\
\hline Machinery and equipment & 145.9 & 176.3 & 157.5 & 206.2 & 248.6 & 212.8 & 337.5 & 216.3 & 218.7 & 248.5 & 421.9 & 298.8 & 348.5 \\
\hline Change in stocks & -0.5 & -2.4 & -1.8 & -0.3 & 1.4 & -0.1 & -1.0 & -2.0 & -1.9 & 1.0 & -0.8 & 0.8 & 0.6 \\
\hline Net exports of goods and services & $2,227.5$ & $2,119.5$ & $2,227.0$ & $1,902.5$ & $1,654.9$ & $1,698.6$ & $1,507.9$ & $2,513.7$ & $2,497.1$ & $2,664.1$ & $2,730.3$ & $2,447.2$ & $1,794.2$ \\
\hline Exports of goods and services & $3,266.0$ & $3,306.5$ & $3,397.4$ & $3,102.5$ & $2,849.7$ & $2,953.5$ & $2,946.4$ & $3,774.9$ & $3,762.6$ & $3,969.3$ & $4,392.7$ & $3,846.8$ & $3,376.1$ \\
\hline Imports of goods and services & $1,038.4$ & $1,187.0$ & $1,170.4$ & $1,200.1$ & $1,194.8$ & $1,254.9$ & $1,438.5$ & $1,261.2$ & $1,265.5$ & $1,305.2$ & $1,662.4$ & $1,399.6$ & $1,581.9$ \\
\hline Statistical discrepancy (residual to tally up) & 175.9 & 172.6 & 297.6 & 298.5 & 142.3 & 219.2 & 156.3 & 271.4 & 178.5 & 113.8 & 218.8 & -344.3 & -866.0 \\
\hline
\end{tabular}

Source: Department of Economic Planning and Development (JPKE). 
Table 5. Brunei Darussalam: Composition of Nominal GDP by Economic Activity, 2002-08 (In percent of total)

\begin{tabular}{|c|c|c|c|c|c|c|c|}
\hline & 2002 & 2003 & 2004 & 2005 & 2006 & 2007 & 2008 \\
\hline Gross domestic product (GDP) & 100.0 & 100.0 & 100.0 & 100.0 & 100.0 & 100.0 & 100.0 \\
\hline Oil and gas sector & 52.9 & 57.2 & 61.9 & 66.4 & 68.5 & 66.8 & 70.1 \\
\hline Oil and gas mining & 40.4 & 44.5 & 50.7 & 55.9 & 59.1 & 57.4 & 57.2 \\
\hline Manufacture of liquefied natural gas (LNG) & 12.5 & 12.7 & 11.2 & 10.5 & 9.5 & 9.4 & 12.9 \\
\hline Non-oil and gas sector & 47.1 & 42.8 & 38.1 & 33.6 & 31.5 & 33.2 & 29.9 \\
\hline Vegetables, fruits and other agriculture & 0.2 & 0.2 & 0.2 & 0.1 & 0.1 & 0.1 & 0.1 \\
\hline Livestock and poultry & 0.3 & 0.3 & 0.3 & 0.2 & 0.2 & 0.2 & 0.2 \\
\hline Forestry & 0.0 & 0.0 & 0.0 & 0.0 & 0.0 & 0.0 & 0.0 \\
\hline Fishery & 0.5 & 0.7 & 0.6 & 0.5 & 0.4 & 0.3 & 0.2 \\
\hline Manufacture of wearing apparel \& textile & 2.4 & 1.9 & 1.4 & 1.2 & 0.7 & 0.6 & 0.5 \\
\hline Other manufacturing & 0.8 & 0.8 & 0.7 & 0.5 & 0.4 & 0.4 & 0.3 \\
\hline Electricity and water & 0.9 & 0.9 & 0.7 & 0.7 & 0.6 & 0.6 & 0.6 \\
\hline Construction & 12.1 & 12.5 & 12.9 & 12.0 & 3.0 & 2.9 & 2.6 \\
\hline Wholesale and retail trade & 3.9 & 3.6 & 3.3 & 2.9 & 2.7 & 3.0 & 2.7 \\
\hline Water transport & 1.0 & 1.2 & 1.0 & 0.9 & 0.9 & 0.8 & 0.7 \\
\hline Air transport & 1.1 & 1.0 & 0.9 & 0.7 & 0.6 & 0.6 & 0.6 \\
\hline Other transport services & 0.3 & 0.3 & 0.2 & 0.3 & 0.4 & 0.5 & 0.5 \\
\hline Communication & 1.6 & 1.5 & 1.3 & 1.0 & 0.8 & 0.8 & 0.8 \\
\hline Finance & 3.4 & 3.1 & 2.8 & 3.0 & 3.0 & 3.1 & 2.9 \\
\hline Real estate and ownership of dwellings & 3.9 & 3.5 & 3.1 & 2.6 & 2.3 & 2.3 & 2.1 \\
\hline Hotels and restaurants & 0.6 & 0.6 & 0.5 & 0.4 & 0.4 & 0.3 & 0.3 \\
\hline Private health and education services & 0.5 & 0.5 & 0.5 & 0.7 & 0.6 & 0.6 & 0.5 \\
\hline Business services & 4.6 & 3.8 & 3.3 & 2.9 & 2.9 & 3.2 & 3.0 \\
\hline Domestic services & 0.5 & 0.4 & 0.4 & 0.3 & 0.3 & 0.3 & 0.3 \\
\hline Other private services & 0.6 & 0.5 & 0.3 & 0.3 & 0.2 & 0.3 & 0.3 \\
\hline Government services & 16.3 & 14.8 & 13.4 & 11.6 & 11.0 & 12.1 & 10.6 \\
\hline
\end{tabular}

Source: Department of Economic Planning and Development (JPKE). 
Table 6. Brunei Darussalam: Quarterly Composition of Nominal GDP by Economic Activity, 2006-09

(In percent of total)

\begin{tabular}{|c|c|c|c|c|c|c|c|c|c|}
\hline & \multicolumn{4}{|c|}{2007} & \multicolumn{4}{|c|}{2008} & \multirow{2}{*}{$\frac{2009}{\mathrm{Q} 1}$} \\
\hline & Q1 & Q2 & Q3 & $\overline{\text { Q4 }}$ & Q1 & Q2 & Q3 & $\overline{\text { Q4 }}$ & \\
\hline Gross domestic product (GDP) & 100.0 & 100.0 & 100.0 & 100.0 & 100.0 & 100.0 & 100.0 & 100.0 & 100.0 \\
\hline Oil and gas sector & 62.7 & 65.9 & 67.1 & 70.4 & 60.2 & 71.5 & 72.7 & 64.6 & 58.0 \\
\hline Oil and gas mining & 53.9 & 56.6 & 58.0 & 60.4 & 62.4 & 62.2 & 62.0 & 40.4 & 39.8 \\
\hline Manufacture of liquefied natural gas (LNG) & 8.8 & 9.4 & 9.1 & 10.0 & 8.4 & 9.3 & 10.7 & 24.3 & 18.2 \\
\hline Non-oil and gas sector & 37.3 & 34.1 & 32.9 & 29.5 & 29.1 & 28.5 & 27.3 & 35.4 & 42.0 \\
\hline Vegetables, fruits \& other agriculture & 0.1 & 0.1 & 0.2 & 0.1 & 0.1 & 0.1 & 0.1 & 0.2 & 0.1 \\
\hline Livestock and poultry & 0.2 & 0.2 & 0.2 & 0.2 & 0.2 & 0.2 & 0.2 & 0.3 & 0.5 \\
\hline Forestry & 0.0 & 0.0 & 0.0 & 0.0 & 0.0 & 0.0 & 0.0 & 0.0 & 0.0 \\
\hline Fishery & 0.3 & 0.3 & 0.3 & 0.3 & 0.2 & 0.2 & 0.2 & 0.3 & 0.3 \\
\hline Manufacture of wearing apparel $\&$ textile & 0.8 & 0.7 & 0.5 & 0.6 & 0.5 & 0.4 & 0.4 & 0.4 & 0.6 \\
\hline Other manufacturing & 0.5 & 0.5 & 0.3 & 0.2 & 0.3 & 0.4 & 0.3 & 0.2 & 0.6 \\
\hline Electricity and water & 0.7 & 0.6 & 0.7 & 0.5 & 0.6 & 0.5 & 0.5 & 0.7 & 0.8 \\
\hline Construction & 2.8 & 2.6 & 3.8 & 2.4 & 2.4 & 2.1 & 3.1 & 2.9 & 3.4 \\
\hline Wholesale and retail trade & 3.6 & 3.2 & 3.1 & 2.2 & 2.9 & 2.8 & 2.5 & 2.7 & 4.4 \\
\hline Water transport & 1.2 & 0.5 & 0.9 & 0.7 & 1.0 & 0.4 & 0.7 & 0.8 & 1.5 \\
\hline Air transport & 0.7 & 0.7 & 0.8 & 0.4 & 0.6 & 0.5 & 0.7 & 0.4 & 0.9 \\
\hline Other transport services & 0.9 & 0.7 & 0.3 & 0.2 & 0.9 & 0.7 & 0.3 & 0.3 & 1.3 \\
\hline Communication & 0.4 & 0.6 & 1.6 & 0.7 & 0.3 & 0.5 & 1.3 & 1.0 & 0.5 \\
\hline Finance & 2.7 & 3.0 & 3.4 & 3.4 & 2.3 & 2.5 & 2.9 & 4.1 & 3.4 \\
\hline Real estate \& ownership of dwellings & 2.5 & 2.5 & 2.4 & 2.0 & 2.1 & 2.1 & 1.9 & 2.3 & 3.0 \\
\hline Hotels and restaurants & 0.4 & 0.4 & 0.3 & 0.3 & 0.3 & 0.3 & 0.2 & 0.3 & 0.4 \\
\hline Private health and education services & 0.7 & 0.7 & 0.5 & 0.4 & 0.6 & 0.4 & 0.4 & 0.5 & 1.0 \\
\hline Business services & 3.6 & 4.7 & 2.1 & 2.8 & 3.1 & 3.9 & 1.9 & 3.5 & 4.4 \\
\hline Domestic services & 0.3 & 0.3 & 0.3 & 0.2 & 0.3 & 0.3 & 0.2 & 0.3 & 0.4 \\
\hline Other private services & 0.2 & 0.4 & 0.3 & 0.2 & 0.2 & 0.3 & 0.3 & 0.2 & 0.2 \\
\hline Government Services & 14.6 & 11.4 & 10.9 & 11.8 & 9.9 & 9.7 & 9.0 & 14.0 & 14.4 \\
\hline
\end{tabular}

Source: Department of Economic Planning and Development (JPKE). 
Table 7. Brunei Darussalam: Composition of Nominal GDP by Expenditure, 2002-08

(In percent of total)

\begin{tabular}{|c|c|c|c|c|c|c|c|}
\hline & 2002 & 2003 & 2004 & 2005 & 2006 & 2007 & 2008 \\
\hline Gross domestic product (GDP) & 100.0 & 100.0 & 100.0 & 100.0 & 100.0 & 100.0 & 100.0 \\
\hline Domestic demand & 74.1 & 66.5 & 62.2 & 52.2 & 48.3 & 55.8 & 48.5 \\
\hline Consumption & 52.8 & 51.4 & 48.7 & 40.8 & 37.9 & 42.8 & 34.8 \\
\hline Personal consumption expenditure & 25.6 & 27.3 & 26.6 & 22.4 & 19.8 & 20.2 & 17.7 \\
\hline Government consumption expenditure & 27.2 & 24.1 & 22.1 & 18.4 & 18.1 & 22.6 & 17.1 \\
\hline Gross capital formation & 21.3 & 15.1 & 13.5 & 11.4 & 10.4 & 13.0 & 13.7 \\
\hline Gross fixed investment & 21.2 & 15.0 & 13.4 & 11.4 & 10.5 & 13.0 & 13.7 \\
\hline Construction & 11.0 & 9.6 & 8.7 & 7.6 & 6.7 & 7.5 & 7.8 \\
\hline Machinery and equipment & 10.2 & 5.4 & 4.7 & 3.7 & 3.8 & 5.5 & 5.8 \\
\hline Change in stocks & 0.1 & 0.1 & 0.1 & 0.0 & 0.0 & 0.0 & 0.0 \\
\hline Net exports of goods and services & 25.5 & 33.3 & 35.7 & 42.9 & 46.5 & 40.0 & 50.7 \\
\hline Exports of goods and services & 67.1 & 69.3 & 68.8 & 70.2 & 71.7 & 67.9 & 78.3 \\
\hline Imports of goods and services & 41.6 & 36.0 & 33.1 & 27.3 & 25.2 & 27.9 & 27.6 \\
\hline Statistical discrepancy (residual to tally up) & 0.5 & 0.2 & 2.2 & 5.0 & 5.2 & 4.3 & 0.8 \\
\hline
\end{tabular}

Source: Department of Economic Planning and Development (JPKE). 
Table 8. Brunei Darussalam: Quarterly Composition of Nominal GDP by Expenditure, 2006-09

(In percent of total)

\begin{tabular}{|c|c|c|c|c|c|c|c|c|c|c|c|c|c|}
\hline & \multicolumn{4}{|c|}{2006} & \multicolumn{4}{|c|}{2007} & \multicolumn{4}{|c|}{2008} & \multirow{2}{*}{$\begin{array}{r}2009 \\
\text { Q1 }\end{array}$} \\
\hline & Q1 & Q2 & Q3 & $\overline{\text { Q4 }}$ & Q1 & Q2 & Q3 & $\overline{\text { Q4 }}$ & Q1 & Q2 & Q3 & $\overline{\mathrm{Q} 4}$ & \\
\hline Gross domestic product (GDP) & 100.0 & 100.0 & 100.0 & 100.0 & 100.0 & 100.0 & 100.0 & 100.0 & 100.0 & 100.0 & 100.0 & 100.0 & 100.0 \\
\hline Domestic demand & 48.2 & 48.8 & 46.7 & 49.7 & 57.5 & 55.2 & 63.0 & 48.9 & 47.4 & 45.2 & 47.3 & 54.8 & 74.0 \\
\hline Consumption & 38.8 & 38.3 & 36.7 & 37.7 & 44.9 & 44.0 & 46.8 & 36.9 & 34.9 & 34.5 & 32.2 & 38.4 & 52.3 \\
\hline Personal consumption expenditure & 18.7 & 21.4 & 20.4 & 18.6 & 20.9 & 23.0 & 22.2 & 15.7 & 17.1 & 18.0 & 16.9 & 19.0 & 25.5 \\
\hline Government consumption expenditure & 20.0 & 16.9 & 16.3 & 19.1 & 24.0 & 21.1 & 24.6 & 21.2 & 17.8 & 16.4 & 15.3 & 19.4 & 26.7 \\
\hline Gross capital formation & 9.4 & 10.5 & 10.0 & 11.9 & 12.7 & 11.2 & 16.1 & 12.1 & 12.5 & 10.8 & 15.1 & 16.4 & 21.7 \\
\hline Gross fixed investment & 9.4 & 10.5 & 10.0 & 12.0 & 12.6 & 11.2 & 16.2 & 12.1 & 12.5 & 10.7 & 15.1 & 16.4 & 21.7 \\
\hline Construction & 6.3 & 6.6 & 6.7 & 7.2 & 6.8 & 6.2 & 8.7 & 8.1 & 8.2 & 5.8 & 7.6 & 10.0 & 11.9 \\
\hline Machinery and equipment & 3.1 & 3.9 & 3.3 & 4.7 & 5.9 & 5.0 & 7.5 & 4.0 & 4.3 & 4.9 & 7.5 & 6.4 & 9.8 \\
\hline Change in stocks & 0.0 & -0.1 & 0.0 & 0.0 & 0.0 & 0.0 & 0.0 & 0.0 & 0.0 & 0.0 & 0.0 & 0.0 & 0.0 \\
\hline Net exports of goods and services & 48.0 & 47.3 & 47.0 & 43.5 & 39.1 & 39.7 & 33.5 & 46.1 & 49.1 & 52.5 & 48.8 & 52.6 & 50.3 \\
\hline Exports of goods and services & 70.4 & 73.8 & 71.7 & 71.0 & 67.4 & 69.0 & 65.6 & 69.2 & 74.0 & 78.3 & 78.5 & 82.7 & 94.7 \\
\hline Imports of goods and services & 22.4 & 26.5 & 24.7 & 27.4 & 28.2 & 29.3 & 32.0 & 23.1 & 24.9 & 25.7 & 29.7 & 30.1 & 44.4 \\
\hline Statistical discrepancy (residual to tally up) & 3.8 & 3.9 & 6.3 & 6.8 & 3.4 & 5.1 & 3.5 & 5.0 & 3.5 & 2.2 & 3.9 & -7.4 & -24.3 \\
\hline
\end{tabular}

Source: Department of Economic Planning and Development (JPKE). 
Table 9. Brunei Darussalam: Real GDP by Economic Activity, 2002-08

(In millions of Brunei dollars, 2000 prices)

\begin{tabular}{|c|c|c|c|c|c|c|c|}
\hline & 2002 & 2003 & 2004 & 2005 & 2006 & 2007 & 2008 \\
\hline Gross domestic product (GDP) & $11,041.5$ & $11,361.5$ & $11,419.4$ & $11,463.7$ & $11,967.8$ & $11,986.3$ & $11,753.9$ \\
\hline Oil and gas sector & $6,155.8$ & $6,431.7$ & $6,365.3$ & $6,201.3$ & $6,469.9$ & $6,023.7$ & $5,650.5$ \\
\hline Oil and gas mining & $4,765.4$ & $4,941.2$ & $4,905.0$ & $4,773.7$ & $4,977.3$ & $4,577.2$ & $4,150.5$ \\
\hline Manufacture of liquefied natural gas (LNG) & $1,390.4$ & $1,490.5$ & $1,460.3$ & $1,427.6$ & $1,492.6$ & $1,446.5$ & $1,500.0$ \\
\hline Non-oil and gas sector & $4,885.7$ & $4,929.8$ & $5,054.4$ & $5,262.5$ & $5,497.9$ & $5,962.6$ & $6,103.4$ \\
\hline Government & $1,720.4$ & $1,712.2$ & $1,766.0$ & $1,816.5$ & $1,977.6$ & $2,206.5$ & $2,266.8$ \\
\hline Private sector & $3,165.3$ & $3,217.6$ & $3,288.4$ & $3,446.0$ & $3,520.2$ & $3,756.1$ & $3,836.6$ \\
\hline Non-oil and gas sector & $4,885.7$ & $4,929.8$ & $5,054.4$ & $5,262.5$ & $5,497.9$ & $5,962.6$ & $6,103.4$ \\
\hline Vegetables, fruits and other agriculture & 23.9 & 30.0 & 31.7 & 31.3 & 27.3 & 28.6 & 35.3 \\
\hline Livestock and poultry & 40.9 & 44.6 & 51.2 & 43.8 & 49.4 & 50.8 & 52.5 \\
\hline Forestry & 4.2 & 4.4 & 5.1 & 5.3 & 5.4 & 4.9 & 6.6 \\
\hline Fishery & 49.0 & 52.4 & 59.1 & 68.5 & 52.2 & 43.9 & 38.6 \\
\hline Manufacture of wearing apparel and textil€ & 222.2 & 165.1 & 165.9 & 173.5 & 135.0 & 137.0 & 132.1 \\
\hline Other manufacturing & 88.1 & 95.7 & 105.4 & 96.6 & 83.8 & 75.5 & 73.5 \\
\hline Electricity and water & 79.1 & 82.1 & 82.4 & 85.8 & 85.9 & 88.2 & 89.0 \\
\hline Construction & 395.3 & 406.9 & 424.0 & 457.8 & 440.8 & 488.9 & 497.6 \\
\hline Wholesale and retail trade & 435.2 & 461.4 & 475.7 & 486.3 & 498.8 & 558.1 & 561.0 \\
\hline Water transport & 109.2 & 126.6 & 111.0 & 134.2 & 144.5 & 165.1 & 178.1 \\
\hline Air transport & 117.7 & 105.0 & 103.8 & 97.7 & 71.6 & 72.1 & 70.5 \\
\hline Other transport services & 35.9 & 32.3 & 31.0 & 36.9 & 61.9 & 100.4 & 109.3 \\
\hline Communication & 164.4 & 171.9 & 174.2 & 168.6 & 163.3 & 158.8 & 169.9 \\
\hline Finance & 350.3 & 361.0 & 379.8 & 417.4 & 475.6 & 493.5 & 505.7 \\
\hline Real estate and ownership of dwellings & 404.8 & 400.5 & 401.7 & 406.7 & 408.0 & 417.4 & 426.3 \\
\hline Hotels and restaurants & 61.8 & 63.0 & 66.1 & 46.6 & 48.2 & 42.2 & 42.3 \\
\hline Private health and education services & 60.9 & 66.4 & 71.2 & 119.8 & 122.0 & 97.1 & 98.2 \\
\hline Business services & 400.7 & 433.7 & 446.5 & 470.6 & 542.0 & 621.4 & 632.8 \\
\hline Domestic services & 44.8 & 45.8 & 46.8 & 47.5 & 48.1 & 48.4 & 49.0 \\
\hline Other private services & 77.0 & 68.8 & 55.8 & 51.1 & 56.3 & 63.6 & 68.3 \\
\hline Government services & $1,720.4$ & $1,712.2$ & $1,766.0$ & $1,816.5$ & $1,977.6$ & $2,206.5$ & $2,266.8$ \\
\hline
\end{tabular}

Source: Department of Economic Planning and Development (JPKE). 
Table 10. Brunei Darussalam: Quarterly Real GDP by Economic Activity, 2006-09

(In millions of Brunei dollars, 2000 prices)

\begin{tabular}{|c|c|c|c|c|c|c|c|c|c|c|c|c|c|}
\hline & \multicolumn{4}{|c|}{2006} & \multicolumn{4}{|c|}{2007} & \multicolumn{4}{|c|}{2008} & \multirow{2}{*}{$\begin{array}{r}2009 \\
\text { Q1 }\end{array}$} \\
\hline & Q1 & Q2 & Q3 & Q4 & Q1 & Q2 & Q3 & Q4 & Q1 & Q2 & Q3 & Q4 & \\
\hline Gross domestic product (GDP) & $3,109.8$ & $2,819.7$ & $2,978.2$ & $3,060.1$ & $3,080.3$ & $2,826.2$ & $2,940.1$ & $3,139.6$ & $2,909.1$ & $2,692.1$ & $2,910.7$ & $3,242.0$ & $2,828.8$ \\
\hline Oil and gas sector & $1,655.4$ & $1,524.4$ & $1,624.8$ & $1,665.3$ & $1,534.9$ & $1,406.4$ & $1,508.5$ & $1,573.9$ & $1,470.9$ & $1,282.2$ & $1,377.0$ & $1,520.4$ & $1,372.3$ \\
\hline Oil and gas mining & $1,271.2$ & $1,172.8$ & $1,247.1$ & $1,286.3$ & $1,175.4$ & $1,061.1$ & $1,137.6$ & $1,203.1$ & $1,091.7$ & 917.9 & $1,008.7$ & $1,132.2$ & $1,000.2$ \\
\hline Manufacture of liquefied natural gas (LNG) & 384.2 & 351.6 & 377.8 & 379.0 & 359.4 & 345.3 & 371.0 & 370.8 & 379.2 & 364.3 & 368.3 & 388.2 & 372.0 \\
\hline Non-oil and gas sector & $1,454.4$ & $1,295.3$ & $1,353.4$ & $1,394.8$ & $1,545.5$ & $1,419.8$ & $1,431.6$ & $1,565.7$ & $1,438.2$ & $1,409.8$ & $1,533.7$ & $1,721.6$ & $1,456.5$ \\
\hline Government & 548.4 & 424.5 & 481.8 & 523.0 & 602.7 & 483.2 & 488.3 & 632.4 & 489.4 & 480.1 & 556.0 & 741.3 & 498.5 \\
\hline Private sector & 906.0 & 870.8 & 871.6 & 871.8 & 942.8 & 936.6 & 943.3 & 933.3 & 948.8 & 929.7 & 977.7 & 980.3 & 958.0 \\
\hline Non-oil and gas sector & $1,454.4$ & $1,295.3$ & $1,353.4$ & $1,394.8$ & $1,545.5$ & $1,419.8$ & $1,431.6$ & $1,565.7$ & $1,438.2$ & $1,409.8$ & $1,533.7$ & $1,721.6$ & $1,456.5$ \\
\hline Vegetables, fruits and other agriculture & 6.3 & 7.0 & 7.1 & 6.9 & 6.5 & 7.0 & 8.0 & 7.2 & 7.9 & 8.8 & 9.5 & 9.1 & 5.1 \\
\hline Livestock and poultry & 12.0 & 11.7 & 12.6 & 13.1 & 12.8 & 12.4 & 12.9 & 12.7 & 12.6 & 13.0 & 14.4 & 12.5 & 17.9 \\
\hline Forestry & 1.2 & 1.5 & 1.6 & 1.1 & 1.2 & 1.4 & 1.1 & 1.2 & 1.4 & 2.1 & 1.9 & 1.2 & 1.0 \\
\hline Fishery & 15.7 & 13.1 & 13.0 & 10.4 & 11.0 & 10.9 & 10.9 & 11.0 & 10.1 & 9.1 & 9.0 & 10.5 & 10.7 \\
\hline Manufacture of wearing apparel \& textile & 37.4 & 30.5 & 34.8 & 32.2 & 35.2 & 36.3 & 31.0 & 34.4 & 44.2 & 22.5 & 29.7 & 35.5 & 29.3 \\
\hline Other manufacturing & 18.7 & 22.8 & 21.4 & 21.0 & 23.3 & 17.4 & 15.7 & 19.1 & 18.7 & 16.3 & 16.8 & 21.6 & 22.6 \\
\hline Electricity and water & 21.1 & 21.4 & 21.9 & 21.6 & 21.0 & 22.3 & 22.5 & 22.4 & 21.2 & 22.3 & 22.6 & 22.9 & 22.2 \\
\hline Construction & 111.7 & 113.5 & 114.6 & 101.0 & 107.6 & 101.7 & 159.0 & 120.6 & 110.4 & 100.7 & 163.4 & 123.1 & 113.5 \\
\hline Wholesale and retail trade & 142.8 & 109.8 & 128.4 & 117.7 & 154.5 & 131.8 & 149.2 & 122.6 & 150.9 & 136.0 & 145.6 & 128.5 & 153.4 \\
\hline Water transport & 69.1 & 16.6 & 29.8 & 29.0 & 65.3 & 25.9 & 37.7 & 36.2 & 62.4 & 27.9 & 47.0 & 40.9 & 65.1 \\
\hline Air transport & 16.6 & 23.1 & 18.2 & 13.7 & 14.9 & 16.5 & 25.2 & 15.5 & 15.5 & 13.7 & 27.2 & 14.1 & 16.2 \\
\hline Other transport services & 26.1 & 8.3 & 12.1 & 15.4 & 33.1 & 32.3 & 17.6 & 17.4 & 27.2 & 43.3 & 19.8 & 18.9 & 27.2 \\
\hline Communication & 16.7 & 38.0 & 70.5 & 38.1 & 16.8 & 27.9 & 74.4 & 39.7 & 17.8 & 28.6 & 76.0 & 47.5 & 18.0 \\
\hline Finance & 98.5 & 93.1 & 120.9 & 163.1 & 101.5 & 98.3 & 125.3 & 168.3 & 106.2 & 101.6 & 124.9 & 173.0 & 109.4 \\
\hline Real estate and ownership of dwellings & 101.8 & 103.0 & 102.2 & 100.9 & 104.0 & 105.3 & 104.2 & 104.0 & 105.4 & 106.5 & 107.4 & 107.0 & 106.2 \\
\hline Hotels and restaurants & 14.0 & 10.9 & 11.6 & 11.8 & 10.6 & 8.9 & 10.9 & 11.8 & 10.8 & 8.4 & 11.1 & 12.0 & 14.2 \\
\hline Private health and education services & 22.9 & 34.7 & 38.8 & 25.5 & 26.1 & 27.0 & 21.4 & 22.6 & 28.3 & 25.9 & 21.2 & 22.9 & 31.0 \\
\hline Business services & 147.6 & 183.1 & 85.9 & 125.5 & 170.6 & 220.2 & 89.2 & 141.4 & 173.8 & 209.6 & 99.1 & 150.3 & 174.1 \\
\hline Domestic services & 12.0 & 12.1 & 12.0 & 12.0 & 12.1 & 12.1 & 12.1 & 12.1 & 12.2 & 12.3 & 12.3 & 12.3 & 12.3 \\
\hline Other private services & 13.8 & 16.6 & 14.1 & 11.8 & 14.5 & 21.1 & 15.0 & 13.1 & 11.8 & 21.1 & 18.8 & 16.6 & 8.7 \\
\hline Government Services & 548.4 & 424.5 & 481.8 & 523.0 & 602.7 & 483.2 & 488.3 & 632.4 & 489.4 & 480.1 & 556.0 & 741.3 & 498.5 \\
\hline
\end{tabular}

Source: Department of Economic Planning and Development (JPKE).

(CInternational Monetary Fund. Not for Redistribution 
Table 11. Brunei Darussalam: Real GDP by Expenditure, 2002-08

(In millions of Brunei dollars, 2000 prices)

\begin{tabular}{|c|c|c|c|c|c|c|c|}
\hline & 2002 & 2003 & 2004 & 2005 & 2006 & 2007 & 2008 \\
\hline Gross domestic product (GDP) & $11,041.5$ & $11,361.5$ & $11,419.4$ & $11,463.7$ & $11,967.8$ & $11,986.2$ & $11,753.8$ \\
\hline Domestic demand & $7,657.4$ & $7,572.0$ & $8,117.0$ & $8,074.4$ & $8,597.6$ & $9,643.3$ & $9,969.2$ \\
\hline Consumption & $5,557.3$ & $5,909.6$ & $6,407.9$ & $6,357.2$ & $6,856.2$ & $7,439.6$ & $7,475.2$ \\
\hline Personal consumption expenditure & $2,696.2$ & $3,122.4$ & $3,505.3$ & $3,485.5$ & $3,615.5$ & $3,687.9$ & $3,752.7$ \\
\hline Government consumption expenditure & $2,861.2$ & $2,787.2$ & $2,902.5$ & $2,871.7$ & $3,240.7$ & $3,751.7$ & $3,722.5$ \\
\hline Gross capital formation & $2,100.1$ & $1,662.4$ & $1,709.1$ & $1,717.3$ & $1,741.4$ & $2,203.7$ & $2,494.0$ \\
\hline Gross fixed investment & $2,093.4$ & $1,654.2$ & $1,702.8$ & $1,715.8$ & $1,744.7$ & $2,204.8$ & $2,497.3$ \\
\hline Construction & $1,083.1$ & $1,114.8$ & $1,161.6$ & $1,176.0$ & $1,159.8$ & $1,274.8$ & $1,444.6$ \\
\hline Machinery and equipment & $1,010.4$ & 539.4 & 541.3 & 539.9 & 584.9 & 930.0 & $1,052.8$ \\
\hline Change in stocks & 6.7 & 8.2 & 6.3 & 1.4 & -3.2 & -1.1 & -3.4 \\
\hline Net exports of goods and services & $3,132.9$ & $3,651.8$ & $3,722.3$ & $3,234.2$ & $3,334.9$ & $2,002.2$ & $1,016.8$ \\
\hline Exports of goods and services & $7,466.7$ & $7,635.7$ & $7,581.2$ & $7,485.7$ & $7,760.4$ & $7,012.5$ & $6,576.6$ \\
\hline Imports of goods and services & $4,333.8$ & $3,983.9$ & $3,858.9$ & $4,251.5$ & $4,425.5$ & $5,010.3$ & $5,559.8$ \\
\hline Statistical discrepancy (residual to tally up) & 251.2 & 137.7 & -419.9 & 154.5 & 35.3 & 340.7 & 767.9 \\
\hline
\end{tabular}

Source: Department of Economic Planning and Development (JPKE). 
Table 12. Brunei Darussalam: Quarterly Real GDP by Expenditure, 2006-09

(In millions of Brunei dollars, 2000 prices)

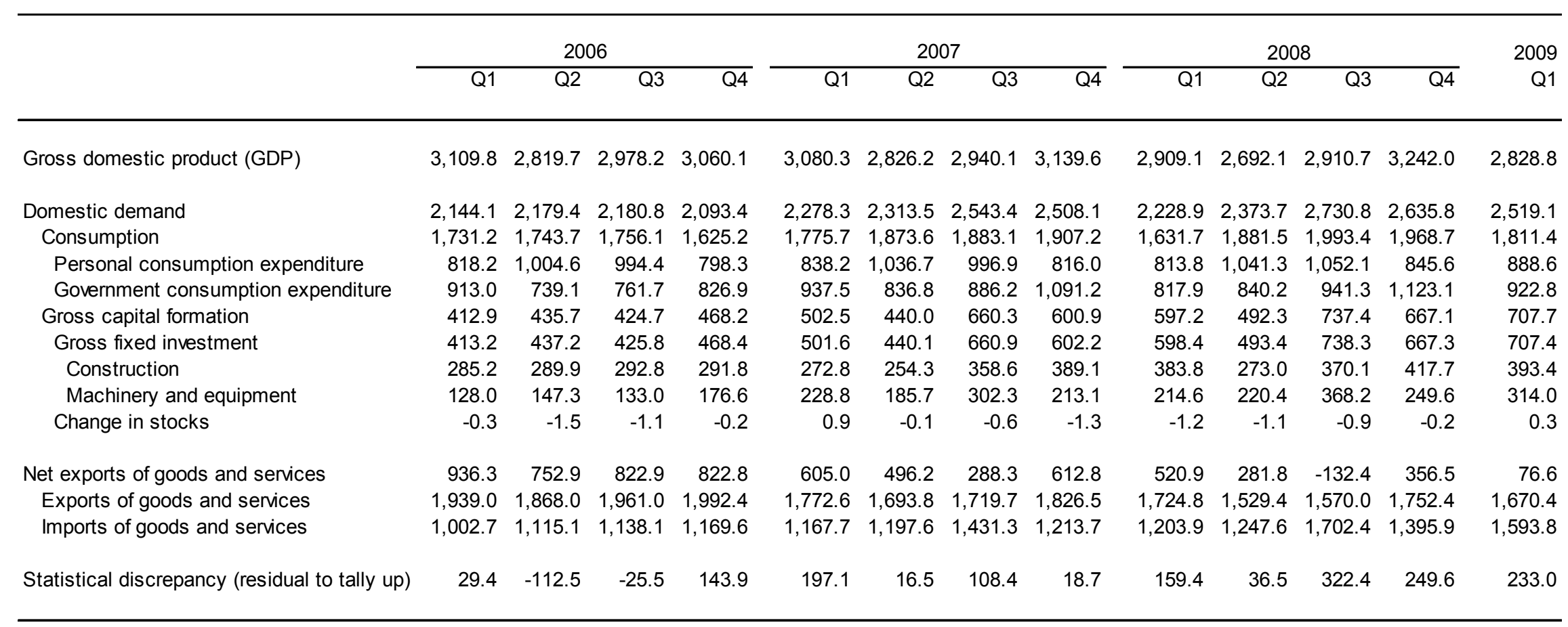

Source: Department of Economic Planning and Development (JPKE). 
Table 13. Brunei Darussalam: Growth in Real GDP by Economic Activity, 2003-08 (Annual percentage change)

\begin{tabular}{|c|c|c|c|c|c|c|}
\hline & 2003 & 2004 & 2005 & 2006 & 2007 & 2008 \\
\hline Gross domestic product (GDP) & 2.9 & 0.5 & 0.4 & 4.4 & 0.2 & -1.9 \\
\hline Oil and gas sector & 4.5 & -1.0 & -2.6 & 4.3 & -6.9 & -6.2 \\
\hline Oil and gas mining & 3.7 & -0.7 & -2.7 & 4.3 & -8.0 & -9.3 \\
\hline Manufacture of liquefied natural gas (LNG) & 7.2 & -2.0 & -2.2 & 4.6 & -3.1 & 3.7 \\
\hline Non-oil and gas sector & 0.9 & 2.5 & 4.1 & 4.5 & 8.5 & 2.4 \\
\hline Vegetables, fruits and other agriculture & 25.5 & 5.7 & -1.3 & -12.8 & 4.8 & 23.4 \\
\hline Livestock and poultry & 9.1 & 14.8 & -14.5 & 12.6 & 2.9 & 3.3 \\
\hline Forestry & 4.8 & 15.9 & 3.9 & 1.0 & -8.5 & 33.4 \\
\hline Fishery & 6.9 & 12.8 & 15.9 & -23.8 & -16.0 & -11.9 \\
\hline Manufacture of wearing apparel and textile & -25.7 & 0.5 & 4.6 & -22.2 & 1.5 & -3.6 \\
\hline Other manufacturing & 8.7 & 10.1 & -8.3 & -13.3 & -9.9 & -2.6 \\
\hline Electricity and water & 3.8 & 0.4 & 4.1 & 0.1 & 2.6 & 0.9 \\
\hline Construction & 2.9 & 4.2 & 8.0 & -3.7 & 10.9 & 1.8 \\
\hline Wholesale and retail trade & 6.0 & 3.1 & 2.2 & 2.6 & 11.9 & 0.5 \\
\hline Water transport & 15.9 & -12.3 & 20.9 & 7.7 & 14.3 & 7.9 \\
\hline Air transport & -10.8 & -1.1 & -5.9 & -26.7 & 0.6 & -2.2 \\
\hline Other transport services & -10.0 & -4.0 & 19.0 & 67.9 & 62.2 & 8.9 \\
\hline Communication & 4.6 & 1.3 & -3.2 & -3.1 & -2.8 & 7.0 \\
\hline Finance & 3.1 & 5.2 & 9.9 & 14.0 & 3.8 & 2.5 \\
\hline Real estate and ownership of dwellings & -1.1 & 0.3 & 1.2 & 0.3 & 2.3 & 2.1 \\
\hline Hotels and restaurants & 1.9 & 4.9 & -29.5 & 3.6 & -12.5 & 0.2 \\
\hline Private health and education services & 9.1 & 7.2 & 68.3 & 1.9 & -20.4 & 1.1 \\
\hline Business services & 8.2 & 3.0 & 5.4 & 15.2 & 14.6 & 1.8 \\
\hline Domestic services & 2.2 & 2.2 & 1.5 & 1.3 & 0.6 & 1.2 \\
\hline Other private services & -10.6 & -18.9 & -8.4 & 10.2 & 13.0 & 7.4 \\
\hline Government services & -0.5 & 3.1 & 2.9 & 8.9 & 11.6 & 2.7 \\
\hline
\end{tabular}

Source: Department of Economic Planning and Development (JPKE). 
Table 14. Brunei Darussalam: Growth in Quarterly Real GDP by Economic Activity, 2006-09

(Annual percentage change)

\begin{tabular}{|c|c|c|c|c|c|c|c|c|c|c|c|c|c|}
\hline & \multicolumn{4}{|c|}{2006} & \multicolumn{4}{|c|}{2007} & \multicolumn{4}{|c|}{2008} & \multirow{2}{*}{$\begin{array}{r}2009 \\
\text { Q1 }\end{array}$} \\
\hline & Q1 & Q2 & Q3 & $\overline{Q 4}$ & Q1 & Q2 & Q3 & $\overline{\mathrm{Q} 4}$ & Q1 & Q2 & Q3 & $\overline{Q 4}$ & \\
\hline Gross domestic product (GDP) & 2.4 & 3.4 & 6.8 & 5.1 & -0.9 & 0.2 & -1.3 & 2.6 & -5.6 & -4.7 & -1.0 & 3.3 & -2.8 \\
\hline Oil and gas sector & 2.5 & 6.2 & 9.4 & 0.1 & -7.3 & -7.7 & -7.2 & -5.5 & -4.2 & -8.8 & -8.7 & -3.4 & -6.7 \\
\hline Oil and gas mining & 2.2 & 4.8 & 9.8 & 0.9 & -7.5 & -9.5 & -8.8 & -6.5 & -7.1 & -13.5 & -11.3 & -5.9 & -8.4 \\
\hline Manufacture of liquefied natural gas (LNG) & 3.3 & 11.3 & 7.9 & -2.7 & -6.4 & -1.8 & -1.8 & -2.2 & 5.5 & 5.5 & -0.7 & 4.7 & -1.9 \\
\hline Non-oil and gas sector & 2.4 & 0.3 & 3.9 & 11.8 & 6.3 & 9.6 & 5.8 & 12.2 & -6.9 & -0.7 & 7.1 & 10.0 & 1.3 \\
\hline Vegetables, fruits and other agriculture & -19.6 & -5.8 & -21.0 & -2.1 & 2.6 & 0.2 & 12.9 & 3.1 & 21.4 & 26.7 & 18.6 & 27.7 & -34.8 \\
\hline Livestock and poultry & 11.0 & 5.9 & 14.1 & 19.5 & 7.3 & 5.9 & 2.5 & -3.1 & -2.1 & 5.1 & 11.6 & -1.3 & 42.4 \\
\hline Forestry & -13.3 & 16.6 & -2.4 & 4.7 & 5.8 & -8.4 & -32.2 & 9.3 & 14.9 & 51.1 & 78.2 & -6.7 & 30.4 \\
\hline Fishery & -46.5 & 18.7 & 13.4 & -37.9 & -29.7 & -16.8 & -15.9 & 5.6 & -8.2 & -17.1 & -17.5 & -4.9 & 5.9 \\
\hline Manufacture of wearing apparel \& textile & -16.0 & -31.6 & -18.5 & -22.4 & -5.9 & 19.0 & -10.8 & 6.7 & 25.6 & -38.0 & -4.2 & 3.3 & -33.7 \\
\hline Other manufacturing & -18.7 & -17.9 & -5.5 & -9.8 & 24.9 & -23.7 & -26.4 & -9.1 & -19.9 & -6.0 & 6.8 & 13.4 & 20.9 \\
\hline Electricity and water & 0.8 & -1.9 & -0.8 & 2.5 & -0.2 & 4.1 & 2.8 & 3.9 & 0.8 & 0.2 & 0.6 & 1.9 & 4.6 \\
\hline Construction & 8.5 & -23.3 & 3.4 & 5.1 & -3.7 & -10.4 & 38.7 & 19.4 & 2.6 & -1.0 & 2.8 & 2.1 & 2.8 \\
\hline Wholesale and retail trade & 8.6 & -8.2 & -0.5 & 11.0 & 8.2 & 20.0 & 16.2 & 4.1 & -2.3 & 3.2 & -2.5 & 4.9 & 1.6 \\
\hline Water transport & 28.5 & 9.8 & -17.1 & -1.3 & -5.5 & 55.6 & 26.3 & 25.1 & -4.5 & 7.8 & 24.7 & 12.8 & 4.4 \\
\hline Air transport & -50.0 & 3.9 & -22.0 & -27.3 & -10.5 & -28.7 & 38.4 & 13.2 & 4.1 & -16.9 & 8.1 & -9.2 & 4.4 \\
\hline Other transport services & 100.9 & -19.2 & 29.8 & 260.4 & 26.7 & 290.7 & 44.9 & 13.4 & -17.8 & 33.9 & 12.7 & 8.6 & -0.2 \\
\hline Communication & -32.4 & 19.5 & -6.2 & 3.2 & 0.5 & -26.6 & 5.6 & 4.3 & 6.2 & 2.4 & 2.1 & 19.6 & 1.00 \\
\hline Finance & 14.4 & 4.4 & 12.2 & 21.5 & 3.0 & 5.6 & 3.6 & 3.2 & 4.6 & 3.4 & -0.3 & 2.7 & 3.0 \\
\hline Real estate and ownership of dwellings & 0.9 & 1.3 & -0.3 & -0.6 & 2.1 & 2.3 & 1.9 & 3.0 & 1.3 & 1.1 & 3.1 & 2.9 & 0.8 \\
\hline Hotels and restaurants & 22.2 & -7.9 & -19.5 & 32.3 & -24.0 & -18.3 & -5.9 & 0.4 & 1.0 & -5.1 & 1.9 & 1.5 & 32.0 \\
\hline Private health and education services & -8.3 & -2.4 & 2.4 & 19.8 & 14.0 & -22.2 & -44.9 & -11.6 & 8.1 & -4.1 & -1.1 & 1.3 & 9.7 \\
\hline Business services & 10.9 & 35.4 & -2.9 & 10.1 & 15.6 & 20.3 & 3.9 & 12.7 & 1.9 & -4.8 & 11.1 & 6.3 & 0.1 \\
\hline Domestic services & 0.0 & 2.1 & 1.6 & 1.6 & 0.6 & 0.6 & 0.6 & 0.6 & 1.2 & 1.2 & 1.4 & 1.5 & 0.2 \\
\hline Other private services & -10.6 & 26.8 & 21.8 & 7.5 & 5.1 & 26.6 & 6.1 & 11.4 & -18.6 & 0.2 & 25.6 & 26.7 & -26.0 \\
\hline Government Services & 1.5 & 1.0 & 13.6 & 21.2 & 9.9 & 13.8 & 1.4 & 20.9 & -18.8 & -0.6 & 13.9 & 17.2 & 1.8 \\
\hline
\end{tabular}

Source: Department of Economic Planning and Development (JPKE).

(CInternational Monetary Fund. Not for Redistribution 
Table 15. Brunei Darussalam: Growth in Real GDP by Expenditure, 2002-08

(Annual percentage change)

\begin{tabular}{|c|c|c|c|c|c|c|c|}
\hline & 2002 & 2003 & 2004 & 2005 & 2006 & 2007 & 2008 \\
\hline Personal consumption expenditure & 0.7 & 15.8 & 12.3 & -0.6 & 3.7 & 2.0 & 1.8 \\
\hline Government consumption expenditure & -2.0 & -2.6 & 4.1 & -1.0 & 12.8 & 15.8 & -0.8 \\
\hline Gross capital formation & 45.7 & -20.8 & 2.8 & 0.5 & 1.4 & 26.5 & 13.2 \\
\hline Gross fixed investment & 43.2 & -21.0 & 2.9 & 0.8 & 1.7 & 26.4 & 13.3 \\
\hline Construction & 9.3 & 2.9 & 4.2 & 1.2 & -1.4 & 9.9 & 13.3 \\
\hline Machinery and equipment & 114.6 & -46.6 & 0.3 & -0.3 & 8.3 & 59.0 & 13.2 \\
\hline Exports of goods and services & 5.8 & 2.3 & -0.7 & -1.3 & 3.7 & -9.6 & -6.2 \\
\hline Exports of goods & 7.9 & 2.5 & -3.0 & -2.9 & 4.9 & -9.1 & -7.5 \\
\hline Exports of services & -9.9 & -0.1 & 20.1 & 10.9 & -4.1 & -13.6 & 3.2 \\
\hline Less: Imports of goods and services & 13.3 & -8.1 & 3.8 & 2.8 & 4.1 & 13.2 & 11.0 \\
\hline Imports of goods & 47.4 & -20.0 & 2.9 & 5.2 & 8.4 & 23.4 & 17.3 \\
\hline Imports of services & -22.2 & 15.4 & 5.0 & -0.3 & -2.0 & -2.8 & -1.7 \\
\hline Gross domestic product (GDP) & 3.9 & 2.9 & 0.5 & 0.4 & 4.4 & 0.2 & -1.9 \\
\hline
\end{tabular}

Source: Department of Economic Planning and Development (JPKE). 
Table 16. Brunei Darussalam: Growth in Quarterly Real GDP by Expenditure, 2006-09

(Annual percentage change)

\begin{tabular}{|c|c|c|c|c|c|c|c|c|c|c|c|c|c|}
\hline & \multicolumn{4}{|c|}{2006} & \multicolumn{4}{|c|}{2007} & \multicolumn{4}{|c|}{2008} & \multirow{2}{*}{$\begin{array}{r}2009 \\
\text { Q1 }\end{array}$} \\
\hline & Q1 & Q2 & Q3 & $\overline{\mathrm{Q} 4}$ & Q1 & Q2 & Q3 & $\overline{\mathrm{Q} 4}$ & Q1 & Q2 & Q3 & $\overline{\mathrm{Q} 4}$ & \\
\hline Gross domestic product (GDP) & 2.4 & 3.4 & 6.8 & 5.1 & -0.9 & 0.2 & -1.3 & 2.6 & -5.6 & -4.7 & -1.0 & 3.3 & -2.8 \\
\hline Domestic demand & 2.9 & -0.9 & 9.8 & 16.0 & 6.3 & 6.2 & 16.6 & 19.8 & -2.2 & 2.6 & 7.4 & 5.1 & 13.0 \\
\hline Consumption & 2.4 & 3.4 & 12.4 & 14.7 & 2.6 & 7.4 & 7.2 & 17.4 & -8.1 & 0.4 & 5.9 & 3.2 & 11.0 \\
\hline Personal consumption expenditure & -2.3 & -1.7 & 11.5 & 8.8 & 2.4 & 3.2 & 0.3 & 2.2 & -2.9 & 0.4 & 5.5 & 3.6 & 9.2 \\
\hline Government consumption expenditure & 6.9 & 11.2 & 13.6 & 21.2 & 2.7 & 13.2 & 16.3 & 32.0 & -12.8 & 0.4 & 6.2 & 2.9 & 12.8 \\
\hline Gross capital formation & 5.3 & -15.1 & 0.3 & 20.5 & 21.7 & 1.0 & 55.5 & 28.3 & 18.8 & 11.9 & 11.7 & 11.0 & 18.5 \\
\hline Gross fixed investment & 5.5 & -14.8 & 0.6 & 20.7 & 21.4 & 0.7 & 55.2 & 28.6 & 19.3 & 12.1 & 11.7 & 10.8 & 18.2 \\
\hline Construction & 7.9 & -23.7 & 2.8 & 18.3 & -4.4 & -12.3 & 22.5 & 33.3 & 40.7 & 7.3 & 3.2 & 7.4 & 2.5 \\
\hline Machinery and equipment & 0.6 & 10.9 & -3.8 & 24.8 & 78.8 & 26.1 & 127.2 & 20.7 & -6.2 & 18.6 & 21.8 & 17.1 & 46.4 \\
\hline Net exports of goods and services & -13.7 & 28.8 & -3.0 & -14.8 & -35.4 & -34.1 & -65.0 & -25.5 & -13.9 & -43.2 & -145.9 & -41.8 & -85.3 \\
\hline Exports of goods and services & 0.4 & 13.3 & 1.9 & 0.5 & -8.6 & -9.3 & -12.3 & -8.3 & -2.7 & -9.7 & -8.7 & -4.1 & -3.1 \\
\hline Imports of goods and services & 4.9 & 5.1 & 1.1 & 5.6 & 16.5 & 7.4 & 25.8 & 3.8 & 3.1 & 4.2 & 19.6 & 15.0 & 32.4 \\
\hline
\end{tabular}

Source: Department of Economic Planning and Development (JPKE). 
Table 17. Brunei Darussalam: Oil and Gas Production and Sales, 2002-08

\begin{tabular}{|c|c|c|c|c|c|c|c|}
\hline & 2002 & 2003 & 2004 & 2005 & 2006 & 2007 & 2008 \\
\hline & \multicolumn{7}{|c|}{ (In thousands of barrels per day) } \\
\hline Oil Production & 203 & 207 & 206 & 201 & 219 & 194 & 174 \\
\hline Crude petroleum & 188 & 193 & 189 & 186 & 204 & 179 & 160 \\
\hline Natural gas liquids & 15 & 14 & 17 & 14 & 16 & 15 & 14 \\
\hline \multirow[t]{2}{*}{ Number of wells (end of year) } & 726 & 722 & 758 & 729 & 738 & 673 & n.a. \\
\hline & \multicolumn{7}{|c|}{ (In billions of cubic meters per year) } \\
\hline Gas production & 11.8 & 12.7 & 12.6 & 12.3 & 12.8 & 12.5 & 12.4 \\
\hline \multicolumn{8}{|l|}{ Export volumes } \\
\hline Oil (thousand of barrels per day) & 199 & 199 & 196 & 193 & 207 & 173 & 153 \\
\hline LNG (trillions of BTU per year) & 352 & 370 & 365 & 357 & 377 & 364 & 366 \\
\hline \multicolumn{8}{|l|}{ Average export prices } \\
\hline Crude oil (US\$/ barrel) & 25.33 & 30.52 & 41.72 & 57.72 & 69.44 & 79.09 & 100.99 \\
\hline LNG (US\$/ MMBtu) & 4.17 & 4.54 & 4.9 & 5.67 & 5.9 & 6.3 & 12.93 \\
\hline
\end{tabular}

Source: Petroleum Unit, Prime Minister's Office. 
Table 18. Brunei Darussaalam: Government Sector Employment by Grade, 2002-08

\begin{tabular}{|c|c|c|c|c|c|c|c|}
\hline & 2002 & 2003 & 2004 & 2005 & 2006 & 2007 & 2008 \\
\hline & \multicolumn{7}{|c|}{ (Number of persons) } \\
\hline Total government employees & 41,066 & 41,505 & 42,191 & 43,469 & 43,515 & 44,777 & 46,091 \\
\hline Division I: Heads of department/section & 599 & 640 & 662 & 711 & 788 & 809 & 903 \\
\hline Division II: Senior Officers & 5,736 & 6,165 & 6,759 & 7,351 & 7,615 & 8,176 & 8,607 \\
\hline Dvision III: Supervisory & 10,400 & 10,269 & 10,266 & 10,206 & 10,167 & 10,620 & 10,828 \\
\hline Division IV: Clerical and operators & 11,644 & 11,651 & 11,571 & 11,818 & 11,617 & 11,734 & 11,996 \\
\hline \multirow[t]{2}{*}{ Division V: Clerical and operators } & 12,687 & 12,780 & 12,933 & 13,383 & 13,328 & 13,438 & 13,757 \\
\hline & \multicolumn{7}{|c|}{ (In percent of total) } \\
\hline Division I: Heads of department/section & 1.5 & 1.5 & 1.6 & 1.6 & 1.8 & 1.8 & 2.0 \\
\hline Division II: Senior Officers & 14.0 & 14.9 & 16.0 & 16.9 & 17.5 & 18.3 & 18.7 \\
\hline Dvision III: Supervisory & 25.3 & 24.7 & 24.3 & 23.5 & 23.4 & 23.7 & 23.5 \\
\hline Division IV: Clerical and operators & 28.4 & 28.1 & 27.4 & 27.2 & 26.7 & 26.2 & 26.0 \\
\hline \multirow[t]{2}{*}{ Division V: Clerical and operators } & 30.9 & 30.8 & 30.7 & 30.8 & 30.6 & 30.0 & 29.8 \\
\hline & \multicolumn{7}{|c|}{ (Percent change) } \\
\hline Total government employees & 3.5 & 1.1 & 1.7 & 3.0 & 0.1 & 2.9 & 2.9 \\
\hline
\end{tabular}

Source: Department of Economic Planning and Development (JPKE). 
Table 19. Brunei Darussalam: Private Sector Employment by Industry and Residency Satus, 2008

\begin{tabular}{|c|c|c|c|c|}
\hline Industry & $\begin{array}{c}\text { Total } \\
\text { Employees }\end{array}$ & $\begin{array}{l}\text { Employees as } \\
\text { Percentage of } \\
\text { Total Employment } \\
\text { (In percent) }\end{array}$ & $\begin{array}{c}\text { Citizens and } \\
\text { Permanent } \\
\text { Residents }\end{array}$ & $\begin{array}{l}\text { Temporary } \\
\text { Residents }\end{array}$ \\
\hline Agriculture, forestry and fishery & 5,156 & 4.1 & 1,480 & 3,676 \\
\hline Production of oil and liquified natural gas & 5,252 & 4.2 & 3,665 & 1,587 \\
\hline Sawmiling and timber processing & 719 & 0.6 & 117 & 602 \\
\hline Other mining, quarrying and manufacturing & 17,626 & 14.1 & 3,202 & 14,424 \\
\hline Construction & 37,151 & 29.6 & 4,881 & 32,270 \\
\hline Wholesale and retail trade & 19,367 & 15.4 & 7,674 & 11,593 \\
\hline Coffee shops, restaurants and hotels & 11,768 & 9.4 & 3,510 & 8,258 \\
\hline Transport, storage and communication & 5,947 & 4.7 & 3,036 & 2,911 \\
\hline Financial, insurance and business services & 9,157 & 7.3 & 6,441 & 2,716 \\
\hline Othe community, social and personal services activities & 13,290 & 10.6 & 4,825 & 8,465 \\
\hline Total private sector employment (all Industries) & 125,333 & 100 & 39,831 & 86,502 \\
\hline
\end{tabular}

Source: Labour Department, Annual Census on Employers and Employees 2008, Ministry of Home Affairs. 
Table 20. Brunei Darussalam: Consumer Price Index, 2006-09

(2002=100; percent change)

\begin{tabular}{|c|c|c|c|c|c|c|}
\hline & \multirow{2}{*}{$\begin{array}{l}\text { Weights } \\
\text { (In percent) }\end{array}$} & \multirow{2}{*}{2006} & \multirow{2}{*}{2007} & \multirow{2}{*}{2008} & \multicolumn{2}{|c|}{2009} \\
\hline & & & & & $\mathrm{H} 1$ & $\mathrm{H} 2$ \\
\hline \multirow[t]{2}{*}{ All groups } & 100.0 & 102.5 & 102.8 & 105.6 & 107.3 & 107.7 \\
\hline & & 0.2 & 0.3 & 2.7 & & \\
\hline \multirow[t]{2}{*}{ Food and non-alcoholic beverages } & 28.8 & 101.6 & 103.8 & 109.0 & 111.4 & 111.5 \\
\hline & & 0.3 & 2.2 & 5.0 & & \\
\hline \multirow[t]{2}{*}{ Clothing and footwear } & 5.6 & 92.2 & 91.5 & 91.8 & 93.0 & 91.9 \\
\hline & & -1.5 & -0.8 & 0.3 & & \\
\hline \multirow[t]{2}{*}{ Housing, water, electricity, and maintenance } & 8.8 & 97.6 & 96.8 & 97.0 & 97.5 & 97.9 \\
\hline & & -1.1 & -0.8 & 0.2 & & \\
\hline \multirow[t]{2}{*}{ Household goods, services, and operation } & 8.6 & 93.1 & 93.9 & 95.5 & 97.4 & 96.2 \\
\hline & & -1.2 & 0.8 & 1.8 & & \\
\hline \multirow[t]{2}{*}{ Transport } & 22.5 & 106.7 & 107.2 & 108.9 & 111.1 & 111.3 \\
\hline & & -1.3 & 0.5 & 1.6 & & \\
\hline \multirow[t]{2}{*}{ Communication } & 5.5 & 88.8 & 88.3 & 88.1 & 87.9 & 87.9 \\
\hline & & -1.7 & -0.6 & -0.2 & & \\
\hline \multirow[t]{2}{*}{ Education } & 4.7 & 98.0 & 97.9 & 98.1 & 97.7 & 97.8 \\
\hline & & -0.3 & -0.1 & 0.2 & & \\
\hline \multirow[t]{2}{*}{ Medical and health } & 1.0 & 100.2 & 101.6 & 102.5 & 102.1 & 102.7 \\
\hline & & 0.1 & 1.4 & 0.9 & & \\
\hline \multirow[t]{2}{*}{ Recreation and entertainment } & 8.1 & 127.2 & 120.9 & 125.9 & 127.8 & 131.9 \\
\hline & & 6.2 & -5.0 & 4.1 & & \\
\hline \multirow[t]{2}{*}{ Miscellaneous goods and services } & 6.4 & 104.0 & 106.8 & 111.1 & 113.2 & 114.3 \\
\hline & & 2.5 & 2.7 & 4.0 & & \\
\hline
\end{tabular}

Source: Brunei Darussalam Statistical Yearbook, Department of Economic Planning and Development. 
Table 21. Brunei Darussalam: Composition of Government Revenue, 2002-2009/10 1/

(In millions of Brunei dollars)

2002 2003/04 2004/05 2005/06 2006/07 2007/08 2007/08 2008/09 2008/09 2009 Budget Outturn Budget Outturn Budget

Actual

Actual

\begin{tabular}{|c|c|c|c|c|c|c|c|c|c|c|}
\hline Total revenue & 4,268 & 6,369 & 6,404 & 8,484 & 9,310 & 5,767 & 10,042 & 5,602 & 11,378 & 4,000 \\
\hline Tax revenue & 2,332 & 3,426 & 3,685 & 5,241 & 5,575 & 3,005 & 6,345 & 3,275 & 7,434 & 2,512 \\
\hline Taxes on net income and profits & 2,209 & 3,283 & 3,563 & 5,099 & 5,452 & 2,869 & 6,176 & 3,137 & 7,275 & 2,367 \\
\hline Corporate taxes & 2,204 & 3,278 & 3,560 & 5,095 & 5,447 & 2,865 & 6,169 & 3,132 & 7,271 & 2,362 \\
\hline Oil and gas production companies & 2,116 & 3,151 & 3,439 & 4,980 & 5,300 & 2,765 & 6,029 & 2,987 & 7,105 & 2,217 \\
\hline Other companies & 89 & 127 & 121 & 116 & 147 & 100 & 140 & 145 & 165 & 145 \\
\hline Individuals (estate duty) & 0 & 1 & 0 & 0 & 1 & 0 & 0 & 1 & 0 & 1 \\
\hline Stamp duty & 5 & 4 & 3 & 4 & 4 & 4 & 6 & 4 & 4 & 4 \\
\hline Taxes on international trade & 111 & 127 & 108 & 125 & 104 & 119 & 151 & 120 & 140 & 126 \\
\hline Motor vehicles tax & 58 & 70 & 62 & 64 & 50 & 64 & 68 & 64 & 76 & 64 \\
\hline Tobacco & 19 & 25 & 21 & 22 & 22 & 22 & 23 & 22 & 21 & 22 \\
\hline Others & 34 & 32 & 25 & 39 & 33 & 33 & 60 & 34 & 43 & 40 \\
\hline Export taxes & & & & & & & & 0 & 0 & 0 \\
\hline Taxes on goods and services & 12 & 16 & 14 & 16 & 18 & 17 & 19 & 19 & 19 & 19 \\
\hline Licenses & 12 & 16 & 14 & 16 & 18 & 17 & 19 & 19 & 19 & 19 \\
\hline Financial companies & 1 & 1 & 1 & 1 & 2 & 2 & 2 & 2 & 1 & 2 \\
\hline Road tax & & & & & & 9 & 9 & 10 & 10 & 10 \\
\hline Brunei International Financal Center & & & & & & 3 & 5 & 5 & 4 & 5 \\
\hline Others & 10 & 15 & 13 & 15 & 16 & 4 & 3 & 3 & 3 & 3 \\
\hline Nontax revenue & 1,936 & 2,943 & 2,719 & 3,243 & 3,735 & 2,762 & 3,696 & 2,327 & 3,944 & 1,488 \\
\hline Property income & 1,631 & 2,569 & 2,419 & 2,880 & 3,465 & 2,450 & 3,235 & 2,036 & 3,669 & 1,168 \\
\hline Oil sector & 1,582 & 2,398 & 2,376 & 2,796 & 3,302 & 2,402 & 2,812 & 1,911 & 3,446 & 1,017 \\
\hline Oil and gas royalties & 384 & 634 & 662 & 854 & 942 & 900 & 992 & 607 & 1,089 & 400 \\
\hline Dividend paid by oil companies & 1,198 & 1,764 & 1,714 & 1,941 & 2,360 & 1,502 & 1,820 & 1,304 & 2,356 & 617 \\
\hline Other & 49 & 171 & 43 & 84 & 163 & 48 & 422 & 125 & 223 & 151 \\
\hline Other royalties & 2 & 2 & 1 & 1 & 2 & 2 & 1 & 2 & 2 & 2 \\
\hline Rent and interest & 25 & 23 & 25 & 52 & 90 & 27 & 377 & 88 & 175 & 114 \\
\hline Others & 22 & 146 & 17 & 31 & 71 & 19 & 44 & 36 & 46 & 36 \\
\hline \multicolumn{10}{|l|}{ Administrative fees and charges on } & 315 \\
\hline Telecom and utilities & 205 & 267 & 217 & 275 & 159 & 219 & 343 & 180 & 154 & 195 \\
\hline Other & 97 & 103 & 79 & 86 & 109 & 90 & 114 & 108 & 117 & 120 \\
\hline Other nontax revenue & 3 & 4 & 3 & 3 & 3 & 3 & 4 & 4 & 4 & 5 \\
\hline \multicolumn{11}{|l|}{ Memorandum items: } \\
\hline Oil and gas sector revenue & 3,697 & 5,550 & 5,815 & 7,775 & 8,602 & 5,167 & 8,841 & 4,898 & 10,551 & 3,234 \\
\hline Non-oil revenue & 571 & 820 & 589 & 709 & 708 & 600 & 1,200 & 704 & 827 & 766 \\
\hline Oil and gas as a percent of total revenue & 87 & 87 & 91 & 92 & 92 & 90 & 88 & 87 & 93 & 81 \\
\hline
\end{tabular}

Sources: Data provided by the Brunei authorities; and Fund staff estimates.

1/ From 2004, Brunei's fiscal year changed from a calendar year to April-March. 2003/04 financial year is from January 2003 to March 2004. 
Table 22. Brunei Darussalam: Composition of Quarterly Government Revenue, 2005/06-2009/10

(In millions of Brunei dollars)

\begin{tabular}{|c|c|c|c|c|c|c|c|c|c|c|c|c|c|c|c|c|c|c|c|}
\hline & \multicolumn{4}{|c|}{$2005 / 06$} & \multicolumn{4}{|c|}{$2006 / 07$} & \multicolumn{4}{|c|}{$2007 / 08$} & \multicolumn{4}{|c|}{$2008 / 09$} & \multicolumn{3}{|c|}{$2009 / 10$} \\
\hline & Jun. & Sep. & Dec. & Mar. & Jun. & Sep. & Dec. & Mar. & Jun. & Sep. & Dec. & Mar. & Jun. & Sep. & Dec. & Mar. & $\begin{array}{l}\text { Jun. } \\
\text { Prov. }\end{array}$ & $\begin{array}{l}\text { Sep. } \\
\text { Prov. }\end{array}$ & $\begin{array}{l}\text { Dec. } \\
\text { Prov. }\end{array}$ \\
\hline Total revenue & 1,770 & 2,276 & 2,192 & 2,245 & 2,511 & 2,603 & 2,261 & 1,935 & 2,075 & 2,281 & 2,482 & 3,203 & 2,778 & 3,879 & 2,349 & 2,371 & 1,461 & 1,516 & 1,236 \\
\hline Tax revenue & 1,006 & 1,444 & 1,488 & 1,303 & 1,628 & 1,651 & 1,331 & 965 & 1,297 & 1,548 & 1,721 & 1,780 & 1,823 & 2,711 & 1,651 & 1,248 & 751 & 1,057 & 710 \\
\hline Taxes on net income and profits & 976 & 1,411 & 1,450 & 1,262 & 1,600 & 1,627 & 1,297 & 928 & 1,240 & 1,512 & 1,682 & 1,742 & 1,788 & 2,670 & 1,612 & 1,206 & 716 & 1,012 & 670 \\
\hline Corporate taxes & 975 & 1,410 & 1,449 & 1,261 & 1,599 & 1,626 & 1,296 & 926 & 1,237 & 1,511 & 1,681 & 1,741 & 1,786 & 2,668 & 1,611 & 1,205 & 715 & 1,010 & 668 \\
\hline Oil and gas production companies & 933 & 1,375 & 1,421 & 1,251 & 1,554 & 1,608 & 1,270 & 868 & 1,165 & 1,446 & 1,679 & 1,739 & 1,729 & 2,588 & 1,598 & 1,191 & 605 & 959 & 654 \\
\hline Other companies & 42 & 35 & 29 & 10 & 45 & 18 & 26 & 58 & 72 & 65 & 2 & 2 & 57 & 81 & 13 & 14 & 110 & 52 & 14 \\
\hline Individuals (estate duty) & 0 & 0 & 0 & 0 & 0 & 0 & 0 & 0 & 0 & 0 & 0 & 0 & 0 & 0 & 0 & 0 & 0 & 0 & 1 \\
\hline Stamp duty & 1 & 1 & 1 & 1 & 1 & 1 & 1 & 1 & 3 & 1 & 1 & 1 & 1 & 1 & 1 & 1 & 1 & 2 & 1 \\
\hline Taxes on international trade & 27 & 29 & 32 & 36 & 24 & 21 & 30 & 29 & 53 & 31 & 34 & 33 & 33 & 36 & 34 & 37 & 31 & 39 & 35 \\
\hline Motor vehicles tax & 15 & 16 & 18 & 15 & 11 & 9 & 17 & 13 & 16 & 17 & 16 & 19 & 18 & 19 & 18 & 21 & 13 & 21 & 15 \\
\hline Tobacco & 6 & 6 & 6 & 4 & 5 & 5 & 4 & 8 & 6 & 5 & 6 & 6 & 5 & 4 & 6 & 5 & 6 & 5 & 6 \\
\hline Others & 6 & 7 & 9 & 18 & 8 & 7 & 9 & 8 & 31 & 9 & 12 & 8 & 9 & 12 & 10 & 11 & 12 & 13 & 13 \\
\hline Taxes on goods and services & 3 & 4 & 5 & 4 & 3 & 3 & 4 & 8 & 4 & 4 & 6 & 5 & 3 & 5 & 4 & 5 & 4 & 5 & 5 \\
\hline Licenses & 3 & 4 & 5 & 4 & 3 & 3 & 4 & 8 & 4 & 4 & 6 & 5 & 3 & 5 & 4 & 5 & 4 & 5 & 5 \\
\hline Financial companies & 0 & 0 & 1 & 0 & 0 & 0 & 1 & 1 & 0 & 0 & 1 & 0 & 0 & 0 & 1 & 0 & 0 & 0 & 1 \\
\hline Road tax & & & & & & & & & 2 & 2 & 2 & 3 & 2 & 3 & 2 & 3 & 3 & 3 & 3 \\
\hline Brunei International Financial Center & & & & & & & & & 1 & 1 & 1 & 1 & 1 & 1 & 1 & 1 & 1 & 1 & 0 \\
\hline Others & 3 & 4 & 4 & 4 & 3 & 3 & 3 & 7 & 1 & 1 & 1 & 1 & 0 & 1 & 1 & 1 & 1 & 1 & 1 \\
\hline Nontax revenue & 764 & 832 & 705 & 943 & 883 & 952 & 930 & 970 & 778 & 734 & 761 & 1,424 & 955 & 1,168 & 698 & 1,123 & 710 & 460 & 526 \\
\hline Property income & 679 & 762 & 632 & 808 & 816 & 896 & 863 & 889 & 705 & 668 & 701 & 1,161 & 890 & 1,103 & 629 & 1,047 & 642 & 389 & 451 \\
\hline Oil sector & 653 & 745 & 613 & 784 & 794 & 864 & 828 & 817 & 684 & 656 & 548 & 924 & 878 & 1,071 & 618 & 879 & 630 & 379 & 445 \\
\hline Oil and gas royalties & 179 & 215 & 215 & 246 & 249 & 263 & 225 & 205 & 207 & 233 & 267 & 285 & 278 & 359 & 250 & 202 & 156 & 171 & 185 \\
\hline Dividend paid by oil companies & 474 & 530 & 399 & 538 & 545 & 601 & 603 & 611 & 477 & 423 & 281 & 639 & 599 & 712 & 368 & 677 & 474 & 208 & 260 \\
\hline Other & 26 & 17 & 18 & 24 & 22 & 32 & 35 & 73 & 21 & 12 & 152 & 237 & 13 & 31 & 11 & 168 & 12 & 10 & 7 \\
\hline Other royalties & 0 & 0 & 0 & 0 & 0 & 0 & 0 & 1 & 0 & 0 & 0 & 0 & 0 & 0 & $1-$ & & -- & - & - \\
\hline Rent and interest & 16 & 11 & 10 & 15 & 16 & 20 & 24 & 30 & 11 & 7 & 149 & 210 & 5 & 5 & 8 & 158 & 4 & 4 & 4 \\
\hline Others & 10 & 5 & 8 & 8 & 6 & 13 & 10 & 42 & 10 & 5 & 4 & 26 & 8 & 26 & 2 & 10 & 8 & 6 & 3 \\
\hline \multicolumn{20}{|l|}{ Administrative fees and charges on } \\
\hline sales of goods and fines & 84 & 70 & 73 & 134 & 66 & 55 & 66 & 80 & 72 & 64 & 59 & 262 & 64 & 64 & 68 & 75 & 67 & 70 & 74 \\
\hline Telecom and utilities & 63 & 49 & 52 & 112 & 47 & 37 & 39 & 36 & 37 & 40 & 33 & 233 & 33 & 36 & 40 & 45 & 39 & 40 & 40 \\
\hline Other & 21 & 21 & 21 & 22 & 19 & 18 & 28 & 44 & 35 & 24 & 26 & 29 & 30 & 28 & 28 & 30 & 28 & 30 & 34 \\
\hline Other nontax revenue & 1 & 1 & 1 & 1 & 1 & 1 & 1 & 1 & 1 & 1 & 1 & 1 & 1 & 1 & 1 & 1 & 1 & 1 & 1 \\
\hline \multicolumn{20}{|l|}{ Memorandum items: } \\
\hline Oil and gas sector revenue & 1,586 & 2,120 & 2,034 & 2,0350 & 2,348 & 2,472 & 2,098 & 1,6850 & 1,849 & 2,102 & 2,227 & 2,663 & 2,606 & 3,659 & 2,216 & 2,070 & 1,235 & 1,338 & 1,099 \\
\hline Non-oil revenue & 184 & 157 & 158 & 2100 & 163 & 131 & 163 & 2500 & 226 & 179 & 255 & 540 & 172 & 220 & 133 & 302 & 226 & 178 & 136 \\
\hline Oil and gas as a percent of total revenue & 90 & 93 & 93 & 91 & 93 & 95 & 93 & 87 & 89 & 92 & 90 & 83 & 94 & 94 & 94 & 87 & 85 & 88 & 89 \\
\hline
\end{tabular}


Table 23. Brunei Darussalam: Composition of Government Expenditure and Budget Balance, 2002-2008/09

(In millions of Brunei dollars)

2002 2003/04 2004/05 2005/06 2006/07 2007/08 2007/08 2008/09 2009

Budget Outturn Budget Outturn Budget

Actual Actual

\begin{tabular}{|c|c|c|c|c|c|c|c|c|c|c|}
\hline Total expenditure & 4,794 & 5,748 & 4,937 & 5,153 & 5,770 & 5,300 & 6,020 & 5,455 & 5,975 & 5,655 \\
\hline Current & 3,777 & 4,596 & 4,048 & 4,126 & 4,497 & 3,814 & 4,878 & 3,907 & 4,957 & 4,013 \\
\hline Other charges annually recurrent & 1,231 & 1,751 & 1,389 & 1,431 & 1,538 & 1,406 & 1,776 & 1,467 & 1,689 & 1,556 \\
\hline Charged & 1,226 & 1,060 & 1,219 & 1,199 & 1,353 & 669 & 1,419 & 669 & 1,549 & 669 \\
\hline Pensions & 237 & 288 & 249 & 270 & 292 & 259 & 325 & 259 & 350 & 259 \\
\hline Capital & 1,017 & 1,151 & 889 & 1,026 & 1,273 & 1,487 & 1,142 & 1,548 & 1,018 & 1,642 \\
\hline Other charges special expenditure & 524 & 765 & 509 & 538 & 602 & 587 & 534 & 498 & 423 & 592 \\
\hline Development expenditure & 435 & 360 & 355 & 488 & 671 & 900 & 608 & 1,050 & 595 & 1,050 \\
\hline Investment in public enterprises & 58 & 26 & 25 & 0 & 0 & 0 & 0 & 0 & 0 & 0 \\
\hline Total revenue & 4,268 & 6,369 & 6,404 & 8,484 & 9,310 & 5,767 & 10,042 & 5,602 & 11,378 & 4,000 \\
\hline Total expenditure & 4,794 & 5,748 & 4,937 & 5,153 & 5,770 & 5,300 & 6,020 & 5,455 & 5,975 & 5,655 \\
\hline Primary budget surplus & -526 & 622 & 1,467 & 3,331 & 3,540 & 466 & 4,021 & 147 & 5,403 & $-1,655$ \\
\hline Total revenue excluding royalties & 3,884 & 5,735 & 5,743 & 7,630 & 8,368 & 4,867 & 9,049 & 4,995 & 10,289 & 3,600 \\
\hline Total expenditure excluding royalties & 3,895 & 5,074 & 4,042 & 4,320 & 4,823 & 4,990 & 5,028 & 5,155 & 4,891 & 5,355 \\
\hline Primary budget surplus excluding royalties & -11 & 661 & 1,700 & 3,310 & 3,546 & -124 & 4,021 & -160 & 5,398 & $-1,755$ \\
\hline Non-oil revenue $1 /$ & 571 & 820 & 589 & 709 & 708 & 600 & 1,200 & 704 & 827 & 766 \\
\hline Non-oil expenditure 2/ & 3,895 & 5,074 & 4,042 & 4,320 & 4,823 & 4,990 & 5,028 & 5,155 & 4,891 & 5,355 \\
\hline Non-oil primary balance & $-3,325$ & $-4,254$ & $-3,453$ & $-3,611$ & $-4,115$ & $-4,391$ & $-3,827$ & $-4,451$ & $-4,064$ & $-4,589$ \\
\hline
\end{tabular}

Sources: Data provided by the Brunei authorities; and Fund staff estimates.

1/ Excludes energy sector corporate taxes, royalties, and dividends.

2/ Excludes disbursements of royalties. 
Table 24. Brunei Darussalam: Composition of Quarterly Government Expenditure and Budget Balance, 2005/06-2009/10

(In millions of Brunei dollars)

\begin{tabular}{|c|c|c|c|c|c|c|c|c|c|c|c|c|c|c|c|c|c|c|c|}
\hline & \multicolumn{4}{|c|}{$2005 / 06$} & \multicolumn{4}{|c|}{$2006 / 07$} & \multicolumn{4}{|c|}{$2007 / 08$} & \multicolumn{4}{|c|}{$2008 / 09$} & \multicolumn{3}{|c|}{$2009 / 10$} \\
\hline & Jun. & Sep. & Dec. & Mar. & Jun. & Sep. & Dec. & Mar. & Jun. & Sep. & Dec. & Mar. & Jun. & Sep. & Dec. & Mar. & $\begin{array}{l}\text { Jun. } \\
\text { Prov. }\end{array}$ & $\begin{array}{l}\text { Sep. } \\
\text { Prov. }\end{array}$ & $\begin{array}{l}\text { Dec. } \\
\text { Prov. }\end{array}$ \\
\hline Total expenditure & 879 & 1,218 & 1,214 & 1,841 & 1,182 & 1,250 & 1,340 & 1,998 & 1,184 & 1,437 & 1,379 & 2,020 & 1,213 & 1,430 & 1,484 & 1,848 & 1,043 & 1,634 & 1,498 \\
\hline Current & 740 & 947 & 971 & 1,468 & 1,037 & 1,050 & 1,062 & 1,347 & 1,030 & 1,076 & 1,188 & 1,584 & 1,081 & 1,233 & 1,233 & 1,410 & 909 & 1,172 & 1,198 \\
\hline Wages and salaries & 342 & 342 & 347 & 464 & 350 & 376 & 388 & 492 & 388 & 384 & 511 & 400 & 392 & 400 & 517 & 409 & 390 & 406 & 528 \\
\hline Other charges annually recurrent & 310 & 343 & 330 & 448 & 352 & 341 & 351 & 494 & 333 & 349 & 327 & 767 & 304 & 367 & 364 & 654 & 276 & 452 & 370 \\
\hline Charged & 87 & 262 & 295 & 556 & 336 & 333 & 322 & 362 & 309 & 342 & 350 & 417 & 385 & 466 & 352 & 347 & 243 & 314 & 300 \\
\hline Pensions & 67 & 62 & 69 & 71 & 67 & 68 & 77 & 79 & 84 & 80 & 71 & 90 & 86 & 88 & 76 & 99 & 84 & 107 & 90 \\
\hline Royalties payments & 0 & 179 & 209 & 445 & 239 & 246 & 215 & 246 & 207 & 228 & 259 & 298 & 278 & 359 & 250 & 196 & 156 & 166 & 185 \\
\hline Others & 20 & 21 & 17 & 39 & 30 & 20 & 30 & 37 & 18 & 34 & 20 & 29 & 18 & 18 & 24 & 40 & 17 & 25 & 23 \\
\hline Capital & 140 & 271 & 243 & 373 & 145 & 200 & 278 & 650 & 154 & 362 & 191 & 435 & 132 & 197 & 251 & 438 & 134 & 462 & 300 \\
\hline Other charges special expenditure & 63 & 150 & 127 & 198 & 69 & 51 & 84 & 398 & 46 & 228 & 61 & 199 & 37 & 58 & 77 & 250 & 65 & 219 & 135 \\
\hline $\begin{array}{l}\text { Development expenditure } \\
\text { Investment in public enterprises }\end{array}$ & 77 & 120 & 115 & 176 & 76 & 149 & 194 & 252 & 108 & 134 & 130 & 237 & $\begin{array}{r}94 \\
0\end{array}$ & 139 & 174 & 188 & 69 & 243 & 164 \\
\hline Total revenue & 1,770 & 2,276 & 2,192 & 2,245 & 2,511 & 2,603 & 2,261 & 1,935 & 2,075 & 2,281 & 2,482 & 3,203 & 2,778 & 3,879 & 2,349 & 2,371 & 1,461 & 1,516 & 1,236 \\
\hline Total expenditure & 879 & 1,218 & 1,214 & 1,841 & 1,182 & 1,250 & 1,340 & 1,998 & 1,184 & 1,437 & 1,379 & 2,020 & 1,213 & 1,430 & 1,484 & 1,848 & 1,043 & 1,634 & 1,498 \\
\hline Primary budget surplus & 891 & 1,058 & 979 & 404 & 1,329 & 1,353 & 921 & -63 & 891 & 844 & 1,103 & 1,184 & 1,566 & 2,449 & 865 & 523 & 419 & -118 & -262 \\
\hline Total revenue excluding royalties & 1,591 & 2,061 & 1,978 & 2,000 & 2,262 & 2,340 & 2,036 & 1,729 & 1,868 & 2,048 & 2,215 & 2,918 & 2,500 & 3,520 & 2,099 & 2,169 & 1,305 & 1,345 & 1,051 \\
\hline Total expenditure excluding royalties & 879 & 1,039 & 1,005 & 1,396 & 943 & 1,004 & 1,124 & 1,751 & 977 & 1,209 & 1,120 & 1,721 & 934 & 1,071 & 1,234 & 1,652 & 887 & 1,469 & 1,313 \\
\hline Primary budget surplus excluding royalties & 712 & 1,022 & 972 & 604 & 1,319 & 1,337 & 912 & -22 & 891 & 839 & 1,095 & 1,197 & 1,566 & 2,449 & 865 & 518 & 419 & -124 & -262 \\
\hline Non-oil revenue $1 /$ & 184 & 157 & 158 & 210 & 163 & 131 & 163 & 250 & 226 & 179 & 255 & 540 & 172 & 220 & 133 & 302 & 226 & 178 & 136 \\
\hline Non-oil expenditure 2/ & 879 & 1,039 & 1,005 & 1,396 & 943 & 1,004 & 1,124 & 1,751 & 977 & 1,209 & 1,120 & 1,721 & 934 & 1,071 & 1,234 & 1,652 & 887 & 1,469 & 1,313 \\
\hline Non-oil primary balance & -695 & -883 & -847 & $-1,186$ & -780 & -872 & -962 & $-1,501$ & -751 & $-1,030$ & -864 & $-1,181$ & -762 & -851 & $-1,101$ & $-1,350$ & -660 & $-1,290$ & $-1,177$ \\
\hline
\end{tabular}

Sources: Data provided by the Brunei authorities; and Fund staff estimates.

1/ Excludes energy sector corporate taxes, royalties, and dividends

2/ Excludes disbursements of royalties. 
Table 25. Brunei Darussalam: Brunei Currency and Monetary Board Survey, 2004-09

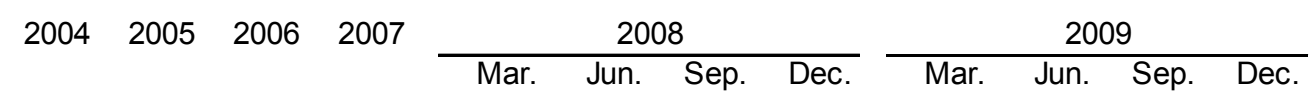

\begin{tabular}{|c|c|c|c|c|c|c|c|c|c|c|c|c|}
\hline \multirow[b]{2}{*}{ Net foreign assets } & \multicolumn{12}{|c|}{ (In millions of Brunei dollars; end of period) } \\
\hline & 794 & 830 & 744 & 963 & 1,006 & 988 & 991 & 1,080 & 1,191 & 1,255 & 1,367 & 1,450 \\
\hline Claims on nonresidents & 822 & 854 & 820 & 998 & 1,040 & 1,023 & 1,043 & 1,115 & 1,223 & 1,287 & 1,857 & 1,938 \\
\hline Foreign exchange & 628 & 718 & 705 & 901 & 948 & 932 & 952 & 1,023 & 1,132 & 1,195 & 1,310 & 1,399 \\
\hline Reserve position in the Fund & 148 & 77 & 57 & 33 & 31 & 30 & 30 & 30 & 31 & 31 & 31 & 30 \\
\hline SDR holdings & 22 & 24 & 26 & 27 & 28 & 28 & 28 & 28 & 29 & 29 & 485 & 476 \\
\hline Other & 23 & 36 & 32 & 36 & 33 & 33 & 33 & 34 & 31 & 32 & 32 & 33 \\
\hline Liabilities to nonresidents & 28 & 24 & 76 & 35 & 34 & 35 & 52 & 35 & 32 & 32 & 490 & 488 \\
\hline Net domestic assets & 533 & 583 & 651 & 494 & 677 & 660 & 715 & 682 & 555 & 447 & 272 & 268 \\
\hline Net claims on central government & -304 & -306 & -293 & -307 & -267 & -267 & -267 & -271 & -269 & -270 & -269 & -268 \\
\hline Claims on central government & 0.33 & 0.33 & 0.60 & 0.51 & 0.50 & 0.65 & 0.67 & 0.65 & 0.74 & 0.70 & 0.84 & 0.86 \\
\hline Central government deposits & 304 & 306 & 294 & 307 & 267 & 267 & 267 & 271 & 270 & 271 & 269 & 269 \\
\hline Claims on other depository corporations & 837 & 888 & 944 & 801 & 944 & 927 & 982 & 953 & 824 & 717 & 540 & 537 \\
\hline Monetary base & 1,263 & 1,377 & 1,395 & 1,459 & 1,446 & 1,472 & 1,493 & 1,691 & 1,666 & 1,623 & 1,546 & 1,624 \\
\hline Currency in circulation & 667 & 705 & 740 & 849 & 801 & 805 & 845 & 922 & 883 & 871 & 883 & 955 \\
\hline Liabilities to other depository corporations & 596 & 672 & 655 & 610 & 645 & 667 & 649 & 769 & 783 & 752 & 663 & 670 \\
\hline Shares and other equity & 86 & 122 & 69 & 88 & 98 & 100 & 102 & 79 & 98 & 99 & 112 & 114 \\
\hline Other items (net) & -23 & -87 & -69 & -90 & -23 & -24 & -23 & -20 & -18 & -19 & -20 & -20 \\
\hline \multicolumn{13}{|l|}{ Memorandum items: } \\
\hline Monetary base (annual growth, in percent) & 9.1 & 9.0 & 1.3 & 4.6 & 11.8 & 15.4 & 12.1 & 15.9 & 15.2 & 10.2 & 3.5 & -4.0 \\
\hline Claims to nonresidents/monetary base (in percent) & 65 & 62 & 59 & 68 & 72 & 69 & 70 & 66 & 73 & 79 & 120 & 119 \\
\hline $\mathrm{BCMB}$ net external asset ratio $1 /$ & 0.94 & 1.02 & 0.95 & 1.06 & 1.18 & 1.16 & 1.13 & 1.11 & 1.28 & 1.37 & 1.48 & 1.47 \\
\hline Prime lending rate (end of period) & 5.50 & 5.50 & 5.50 & 5.50 & 5.50 & 5.50 & 5.50 & 5.50 & 5.50 & 5.50 & 5.50 & 5.50 \\
\hline Brunei dollars per U.S. dollar (end of period) & 1.63 & 1.66 & 1.53 & 1.44 & 1.38 & 1.36 & 1.43 & 1.44 & 1.52 & 1.45 & 1.41 & 1.40 \\
\hline
\end{tabular}

Source: IMF, International Financial Statistics.

$1 /$ Ratio of foreign exchange holding to currency. 
Table 26. Brunei Darussalam: Other Depository Corporations Survey, 2004-09

\begin{tabular}{|c|c|c|c|c|c|c|c|c|c|c|c|c|}
\hline & \multirow[t]{2}{*}{2004} & \multirow[t]{2}{*}{2005} & \multirow[t]{2}{*}{2006} & \multirow[t]{2}{*}{2007} & \multicolumn{4}{|c|}{2008} & \multicolumn{4}{|c|}{2009} \\
\hline & & & & & Mar. & Jun. & Sep. & $\overline{\text { Dec. }}$ & Mar. & Jun. & Sep. & Dec. \\
\hline & \multicolumn{12}{|c|}{ (In millions of Brunei dollars; end of period) } \\
\hline Net foreign assets & 7,096 & 7,793 & 6,886 & 7,179 & 7,204 & 7,394 & 9,096 & 9,468 & 9,247 & 7,113 & 6,916 & 7,128 \\
\hline Claims on nonresidents & 7,244 & 8,291 & 7,418 & 7,691 & 7,582 & 7,800 & 9,672 & 9,847 & 9,617 & 7,552 & 7,335 & 7,542 \\
\hline Liabilities to nonresidents & 148 & 498 & 532 & 512 & 378 & 406 & 576 & 379 & 370 & 438 & 419 & 414 \\
\hline Claims on BCMB & 685 & 774 & 794 & 761 & 772 & 787 & 831 & 955 & 931 & 881 & 810 & 842 \\
\hline Net claims on central government & $-3,226$ & $-4,510$ & $-3,125$ & $-3,270$ & $-2,966$ & $-2,723$ & $-4,318$ & $-5,105$ & $-3,874$ & $-2,151$ & $-1,879$ & $-1,713$ \\
\hline Claims on central government & 54 & 50 & 180 & 129 & 65 & 133 & 113 & 203 & 190 & 167 & 236 & 288 \\
\hline Central government deposits & 3,280 & 4,560 & 3,305 & 3,399 & 3,030 & 2,856 & 4,431 & 5,308 & 4,064 & 2,318 & 2,114 & 2,001 \\
\hline Claims on other sectors & 6,261 & 6,463 & 6,395 & 7,005 & 7,041 & 7,109 & 7,292 & 7,229 & 7,191 & 7,205 & 7,203 & 6,984 \\
\hline Claims on private sector & 6,179 & 6,392 & 6,335 & 6,882 & 6,932 & 7,003 & 7,195 & 7,137 & 7,104 & 7,125 & 7,133 & 6,920 \\
\hline Claims on public nonfinancial corporations & 82 & 71 & 60 & 123 & 109 & 105 & 97 & 92 & 87 & 80 & 69 & 63 \\
\hline Claims on other financial corporations & 0.00 & 0.00 & 0.00 & 0.00 & 0.13 & 0.08 & 0.08 & 0.05 & 0.05 & 0.12 & 0.16 & 0.10 \\
\hline Liabilities to BCMB & 836 & 858 & 936 & 771 & 751 & 831 & 836 & 899 & 800 & 689 & 519 & 484 \\
\hline Tran. dep. included in broad money & 2,955 & 2,975 & 3,321 & 3,154 & 3,403 & 3,185 & 3,239 & 3,647 & 4,024 & 4,076 & 4,583 & 4,607 \\
\hline Other dep. included in broad money & 6,060 & 5,584 & 5,392 & 6,132 & 6,384 & 7,033 & 7,257 & 6,553 & 7,105 & 6,813 & 6,563 & 6,605 \\
\hline Sec. ot. th. shares incl. in brd. money & 2.78 & 3.14 & 1.59 & 0.52 & 0.69 & 0.71 & 0.98 & 1.09 & 1.84 & 0.84 & 0.30 & 1.08 \\
\hline Shares and other equity & 882 & 974 & 1,151 & 1,572 & 1,633 & 1,667 & 1,670 & 1,705 & 1,784 & 1,831 & 1,845 & 1,904 \\
\hline Other items (net) & 79 & 127 & 148 & 46 & -120 & -117 & -93 & -280 & -219 & -362 & -460 & -361 \\
\hline \multicolumn{13}{|l|}{ Memorandum items: } \\
\hline Net foreign assets (annual growth, in percent) & 33.7 & 9.8 & -11.6 & 4.3 & 21.5 & 38.9 & 79.6 & 50.3 & 28.4 & -3.8 & -24.0 & -24.7 \\
\hline Claims on private sector (annual growth, in percent) & 6.4 & 3.5 & -0.9 & 8.6 & 6.4 & 3.8 & 4.5 & 3.7 & 2.5 & 1.7 & -0.8 & -3.0 \\
\hline Prime lending rate (end of period) & 5.50 & 5.50 & 5.50 & 5.50 & 5.50 & 5.50 & 5.50 & 5.50 & 5.50 & 5.50 & 5.50 & 5.50 \\
\hline Brunei dollars per U.S. dollar (end of period) & 1.63 & 1.66 & 1.53 & 1.44 & 1.38 & 1.36 & 1.43 & 1.44 & 1.52 & 1.45 & 1.41 & 1.40 \\
\hline
\end{tabular}

Source: IMF, International Financial Statistics. 
Table 27. Brunei Darussalam: Depository Corporations Survey, 2004-09

\begin{tabular}{|c|c|c|c|c|c|c|c|c|c|c|c|c|}
\hline & \multirow[t]{2}{*}{2004} & \multirow[t]{2}{*}{2005} & \multirow[t]{2}{*}{2006} & \multirow[t]{2}{*}{2007} & \multicolumn{4}{|c|}{2008} & \multicolumn{4}{|c|}{2009} \\
\hline & & & & & Mar. & Jun. & Sep. & $\overline{\text { Dec. }}$ & Mar. & Jun. & Sep. & Dec. \\
\hline & \multicolumn{12}{|c|}{ (In millions of Brunei dollars; end of period) } \\
\hline Net foreign assets & 7,890 & 8,623 & 7,630 & 8,142 & 8,210 & 8,382 & 10,087 & 10,548 & 10,438 & 8,368 & 8,283 & 8,577 \\
\hline Claims on nonresidents & 8,066 & 9,145 & 8,238 & 8,689 & 8,622 & 8,823 & 10,715 & 10,962 & 10,841 & 8,839 & 9,192 & 9,480 \\
\hline Liabilities to nonresidents & 176 & 522 & 608 & 547 & 412 & 440 & 628 & 414 & 402 & 470 & 909 & 902 \\
\hline Domestic claims & 2,731 & 1,647 & 2,977 & 3,428 & 3,808 & 4,118 & 2,707 & 1,853 & 3,048 & 4,784 & 5,055 & 5,002 \\
\hline Net claims on central government & $-3,530$ & $-4,816$ & $-3,418$ & $-3,577$ & $-3,233$ & $-2,990$ & $-4,585$ & $-5,376$ & $-4,143$ & $-2,421$ & $-2,147$ & $-1,981$ \\
\hline Claims on central government & 54.12 & 50.47 & 180.77 & 129.69 & 65.13 & 133.37 & 114.02 & 203.47 & 190.48 & 167.56 & 236.61 & 289.06 \\
\hline Central government deposits & 3,584 & 4,866 & 3,599 & 3,707 & 3,298 & 3,124 & 4,699 & 5,579 & 4,334 & 2,589 & 2,384 & 2,271 \\
\hline Claims on other sectors & 6,261 & 6,463 & 6,395 & 7,005 & 7,041 & 7,109 & 7,292 & 7,229 & 7,191 & 7,205 & 7,203 & 6,984 \\
\hline Claims on private sector & 6,179 & 6,392 & 6,335 & 6,882 & 6,932 & 7,003 & 7,195 & 7,137 & 7,104 & 7,125 & 7,133 & 6,920 \\
\hline Claims on other financial corporations & 0 & 0 & 0 & 0 & 0.13 & 0.08 & 0.08 & 0.05 & 0.05 & 0.12 & 0.16 & 0.10 \\
\hline Reserve money & 1,263 & 1,377 & 1,395 & 1,459 & 1,446 & 1,472 & 1,493 & 1,691 & 1,666 & 1,623 & 1,546 & 1,624 \\
\hline Currency outside depository corp. & 576 & 601 & 641 & 697 & 674 & 686 & 702 & 739 & 731 & 736 & 734 & 784 \\
\hline Transferable deposits & 2,955 & 2,975 & 3,321 & 3,154 & 3,403 & 3,185 & 3,239 & 3,647 & 4,024 & 4,076 & 4,583 & 4,607 \\
\hline Other deposits & 6,060 & 5,584 & 5,392 & 6,132 & 6,384 & 7,033 & 7,257 & 6,553 & 7,105 & 6,813 & 6,563 & 6,605 \\
\hline Securities other than shares & 2.78 & 3.14 & 1.59 & 0.52 & 0.69 & 0.71 & 0.98 & 1.09 & 1.84 & 0.84 & 0.30 & 1.08 \\
\hline Shares and other equity & 968 & 1096 & 1219 & 1660 & 1731 & 1767 & 1772 & 1784 & 1882 & 1929 & 1957 & 2019 \\
\hline Other items (net) & 59 & 12 & 31 & -74 & -174 & -171 & -177 & -324 & -258 & -402 & -498 & -435 \\
\hline \multicolumn{13}{|l|}{ Memorandum items: } \\
\hline Prime lending rate (end of period) & 5.50 & 5.50 & 5.50 & 5.50 & 5.50 & 5.50 & 5.50 & 5.50 & 5.50 & 5.50 & 5.50 & 5.50 \\
\hline Brunei dollars per U.S. dollar (end of period) & 1.63 & 1.66 & 1.53 & 1.44 & 1.38 & 1.36 & 1.43 & 1.44 & 1.52 & 1.45 & 1.41 & 1.40 \\
\hline
\end{tabular}

Source: IMF, International Financial Statistics. 
Table 28. Brunei Darussalam: Direction of Loans by Commercial Banks, 2004-09

\begin{tabular}{|c|c|c|c|c|c|c|c|c|}
\hline & \multirow[t]{2}{*}{2004} & \multirow[t]{2}{*}{2005} & \multirow[t]{2}{*}{2006} & \multirow[t]{2}{*}{2007} & \multirow[t]{2}{*}{2008} & \multicolumn{3}{|c|}{2009} \\
\hline & & & & & & Mar. & Jun. & Sep. \\
\hline & \multicolumn{8}{|c|}{ (In millions of Brunei dollars; end of period) } \\
\hline Total loans & 5,576 & 5,932 & 5,819 & 6,036 & 6,113 & 6,054 & 6,046 & 6,039 \\
\hline Agricultural & 34 & 35 & 30 & 31 & 37 & 37 & 38 & 37 \\
\hline Construction & 496 & 501 & 555 & 495 & 503 & 469 & 494 & 513 \\
\hline Credit and finance & 11 & 14 & 22 & 44 & 29 & 30 & 31 & 33 \\
\hline General commerce & 504 & 533 & 521 & 561 & 603 & 583 & 591 & 587 \\
\hline Manufacturing & 113 & 164 & 180 & 179 & 146 & 150 & 176 & 176 \\
\hline Personal loans & 4,167 & 4,078 & 3,834 & 3,675 & 3,635 & 3,610 & 3,559 & 3,535 \\
\hline Mortgages & 449 & 741 & 820 & 1,014 & 1,190 & 1,207 & 1,204 & 1,227 \\
\hline Other & 3,718 & 3,337 & 3,014 & 2,661 & 2,445 & 2,403 & 2,355 & 2,308 \\
\hline Professional services & 44 & 68 & 81 & 79 & 90 & 88 & 93 & 92 \\
\hline \multirow[t]{2}{*}{ Transport } & 207 & 539 & 596 & 972 & 1,070 & 1,087 & 1,064 & 1,066 \\
\hline & \multicolumn{8}{|c|}{ (In percent of total loans) } \\
\hline Total Ioans & 100.0 & 100.0 & 100.0 & 100.0 & 100.0 & 100.0 & 100.0 & 100.0 \\
\hline Agricultural & 0.6 & 0.6 & 0.5 & 0.5 & 0.6 & 0.6 & 0.6 & 0.6 \\
\hline Construction & 8.9 & 8.4 & 9.5 & 8.2 & 8.2 & 7.7 & 8.2 & 8.5 \\
\hline Credit and finance & 0.2 & 0.2 & 0.4 & 0.7 & 0.5 & 0.5 & 0.5 & 0.5 \\
\hline General commerce & 9.0 & 9.0 & 9.0 & 9.3 & 9.9 & 9.6 & 9.8 & 9.7 \\
\hline Manufacturing & 2.0 & 2.8 & 3.1 & 3.0 & 2.4 & 2.5 & 2.9 & 2.9 \\
\hline Personal loans & 74.7 & 68.7 & 65.9 & 60.9 & 59.5 & 59.6 & 58.9 & 58.5 \\
\hline Mortgages & 8.1 & 12.5 & 14.1 & 16.8 & 19.5 & 19.9 & 19.9 & 20.3 \\
\hline Other & 66.7 & 56.3 & 51.8 & 44.1 & 40.0 & 39.7 & 39.0 & 38.2 \\
\hline Professional services & 0.8 & 1.1 & 1.4 & 1.3 & 1.5 & 1.5 & 1.5 & 1.5 \\
\hline \multirow[t]{2}{*}{ Transport } & 3.7 & 9.1 & 10.2 & 16.1 & 17.5 & 18.0 & 17.6 & 17.7 \\
\hline & \multicolumn{8}{|c|}{ (Annual percentage change) } \\
\hline Total loans & 2.3 & 6.4 & -1.9 & 3.7 & 1.3 & -1.0 & -0.1 & -0.1 \\
\hline Agricultural & 3.0 & 2.9 & -14.3 & 3.3 & 19.4 & 0.0 & 2.7 & -2.6 \\
\hline Construction & 8.2 & 1.0 & 10.8 & -10.8 & 1.6 & -6.8 & 5.3 & 3.8 \\
\hline Credit and finance & -52.2 & 27.3 & 57.1 & 100.0 & -34.1 & 3.4 & 3.3 & 6.5 \\
\hline General commerce & 4.0 & 5.8 & -2.3 & 7.7 & 7.5 & -3.3 & 1.4 & -0.7 \\
\hline Manufacturing & 25.6 & 45.1 & 9.8 & -0.6 & -18.4 & 2.7 & 17.3 & 0.0 \\
\hline Personal loans & 2.8 & -2.1 & -6.0 & -4.1 & -1.1 & -0.7 & -1.4 & -0.7 \\
\hline Mortgages & -6.3 & 65.0 & 10.7 & 23.7 & 17.4 & 1.4 & -0.2 & 1.9 \\
\hline Other & 4.3 & -10.2 & -9.7 & -11.7 & -8.1 & -1.7 & -2.0 & -2.0 \\
\hline Professional services & -56.0 & 54.5 & 19.1 & -2.5 & 13.9 & -2.2 & 5.7 & -1.1 \\
\hline Transport & 3.0 & 160.4 & 10.6 & 63.1 & 10.1 & 1.6 & -2.1 & 0.2 \\
\hline
\end{tabular}

Source: Financial Institutions Division, Ministry of Finance. 
Table 29. Brunei Darussalam: Financial Soudness Indicators, 2007-09

\begin{tabular}{|c|c|c|c|c|c|c|c|c|c|c|c|c|}
\hline & \multicolumn{4}{|c|}{2007} & \multicolumn{4}{|c|}{2008} & \multicolumn{4}{|c|}{2009} \\
\hline & Mar. & Jun. & Sep. & $\overline{\text { Dec. }}$ & Mar. & Jun. & Sep. & $\overline{\text { Dec. }}$ & Mar. & Jun. & Sep. & Dec. \\
\hline & \multicolumn{12}{|c|}{ (Average all banks, in percentages) } \\
\hline \multicolumn{13}{|l|}{ Capital adequacy } \\
\hline Regulatory capital to risk-weighted assets & 7.7 & 7.7 & 6.7 & 7.0 & 8.0 & 8.1 & 7.9 & 14.0 & 14.4 & 15.8 & 15.4 & 18.4 \\
\hline Regulatory tier 1 capital to risk-weighted assets & 5.0 & 5.4 & 5.0 & 5.3 & 6.3 & 6.5 & 6.8 & 13.8 & 13.9 & 15.6 & 15.2 & 19.3 \\
\hline \multicolumn{13}{|l|}{ Asset quality } \\
\hline Net nonperforming ratio $1 /$ & 13.0 & 13.1 & 12.3 & 12.4 & 12.5 & 13.9 & 12.4 & 8.4 & 9.0 & 9.1 & 9.8 & 10.3 \\
\hline Nonperforming loans net of provisions to capital & 11.8 & 10.2 & 10.3 & 10.4 & 12.0 & 11.9 & 12.5 & 1.3 & 1.8 & 2.2 & 2.7 & 3.1 \\
\hline \multicolumn{13}{|l|}{ Sectoral distribution of loans to total loans } \\
\hline Personal Loans & 35.5 & 34.3 & 33.1 & 31.6 & 30.9 & 31.4 & 40.3 & 40.0 & 39.7 & 39.0 & 38.2 & 41.5 \\
\hline Mortgage & 25.5 & 26.3 & 25.6 & 25.3 & 25.4 & 25.8 & 18.8 & 19.5 & 19.9 & 19.9 & 20.3 & 17.5 \\
\hline Transportation & 4.7 & 5.2 & 7.5 & 8.8 & 8.8 & 8.9 & 16.3 & 17.5 & 18.0 & 17.6 & 17.7 & 18.5 \\
\hline General commerce & 11.7 & 12.3 & 12.1 & 12.4 & 12.2 & 11.1 & 9.6 & 9.9 & 9.6 & 9.8 & 9.7 & 10.0 \\
\hline Manufacturing & 5.7 & 5.3 & 5.2 & 5.0 & 5.2 & 4.7 & 4.5 & 2.4 & 2.5 & 2.9 & 2.9 & 2.5 \\
\hline Professional services & 1.7 & 1.7 & 1.4 & 1.3 & 1.3 & 1.3 & 1.4 & 1.5 & 1.5 & 1.5 & 1.5 & 1.6 \\
\hline Agriculture & 3.5 & 3.5 & 3.5 & 3.5 & 0.6 & 0.6 & 0.6 & 0.6 & 0.6 & 0.6 & 0.6 & 0.5 \\
\hline Credit \& financial & 0.7 & 0.7 & 0.5 & 0.5 & 2.1 & 2.9 & 0.4 & 0.5 & 0.5 & 0.5 & 0.5 & 0.6 \\
\hline \multicolumn{13}{|l|}{ Earnings and profitability } \\
\hline Return on assets, before tax & 0.9 & 1.0 & 0.9 & 1.4 & 1.2 & 1.4 & 1.6 & 1.5 & 1.7 & 1.6 & 1.7 & 1.5 \\
\hline Return on equity, after tax & 4.1 & 6.3 & 6.0 & 8.4 & 4.0 & 6.1 & 14.4 & 13.0 & 20.2 & 18.4 & 16.4 & 13.2 \\
\hline Interest margin to gross income & 63.4 & 65.6 & 64.5 & 73.2 & 74.5 & 74.8 & 72.0 & 60.4 & 65.2 & 66.3 & 66.1 & 62.0 \\
\hline Non-interest expenses to gross income & 37.0 & 35.8 & 40.6 & 37.3 & 37.2 & 52.3 & 36.7 & 37.9 & 35.6 & 38.4 & 40.1 & 41.7 \\
\hline \multicolumn{13}{|l|}{ Liquidity } \\
\hline Liquid assets to total assets (liquid asset ratio) & 29.7 & 31.4 & 27.0 & 34.6 & 38.5 & 40.8 & 39.2 & 59.3 & 57.5 & 52.2 & 51.6 & 59.1 \\
\hline Liquid assets to short-term liabilities (demand and savings deposits) & 41.6 & 45.5 & 45.1 & 48.9 & 53.4 & 52.9 & 52.3 & 186.8 & 162.3 & 128.9 & 130.4 & 146.5 \\
\hline \multicolumn{13}{|l|}{ Sensitivity to market risk } \\
\hline Net open position in foreign exchange to capital & 78.6 & 78.1 & 61.6 & 64.1 & 61.8 & 84.7 & 87.3 & $\ldots$ & $\ldots$ & $\ldots$ & $\ldots$ & $\ldots$ \\
\hline
\end{tabular}

Source: Financial Institutions Division, Ministry of Finance.

1/ The calculation of NPL ratio is based on the FSI Compilation Guide (page 183, Appendix II, Para 22). Accrued interest on NPA is excluded from both the numerator and denominator. 
Table 30. Brunei Darussalam: Balance of Payments, 2003-08 1/

(In millions of U.S. dollars; unless otherwise indicated)

\begin{tabular}{|c|c|c|c|c|c|c|}
\hline & 2003 & 2004 & 2005 & 2006 & 2007 & 2008 \\
\hline Current account balance & 3,129 & 3,827 & 5,032 & 6,462 & 6,232 & 8,523 \\
\hline In percent of GDP & 47.7 & 48.6 & 52.8 & 56.3 & 50.9 & 59.1 \\
\hline Trade balance & 3,167 & 3,721 & 4,836 & 6,026 & 5,677 & 8,281 \\
\hline Exports & 4,421 & 5,057 & 6,249 & 7,608 & 7,668 & 10,721 \\
\hline Oil and gas & 3,876 & 4,624 & 5,886 & 7,327 & 7,373 & 10,344 \\
\hline Other 2/ & 545 & 433 & 363 & 281 & 295 & 377 \\
\hline Imports & 1,254 & 1,336 & 1,413 & 1,582 & 1,991 & 2,440 \\
\hline Services (net) & -594 & -530 & -494 & -468 & -503 & -539 \\
\hline Receipts & 435 & 544 & 617 & 742 & 813 & 862 \\
\hline Payments & 1,029 & 1,074 & 1,111 & 1,210 & 1,316 & 1,401 \\
\hline Income (net) 3/ & 845 & 944 & 1,067 & 1,308 & 1,488 & 1,226 \\
\hline Receipts & 1,058 & 1,172 & 1,257 & 1,490 & 1,694 & 1,453 \\
\hline Payments & 213 & 228 & 190 & 182 & 206 & 227 \\
\hline Current transfers (net) & -290 & -309 & -376 & -404 & -430 & -446 \\
\hline Receipts & 0 & 0 & 0 & 0 & 0 & 0 \\
\hline Payments & 290 & 309 & 376 & 404 & 430 & 446 \\
\hline Capital and financial account balance & $-1,469$ & $-2,136$ & $-1,668$ & $-1,616$ & -9 & 14 \\
\hline Capital account balance (net) & -1.2 & -11.3 & -11.5 & -7.0 & -9.3 & -12.8 \\
\hline Financial account balance & $-1,468$ & $-2,125$ & $-1,657$ & $-1,609$ & 1 & 27 \\
\hline Direct investment (net) & 122 & 70 & 167 & 433 & 260 & 237 \\
\hline Portfolio investment (net) & 60 & -45 & 21 & 50 & 161 & 100 \\
\hline Other investment (net) $3 / 4 /$ & $-1,650$ & $-2,150$ & $-1,845$ & $-2,092$ & -421 & -310 \\
\hline Errors and omissions 5/ & $-1,634$ & $-1,677$ & $-3,361$ & $-4,825$ & $-5,576$ & $-8,027$ \\
\hline Overall balance & 26 & 14 & 3 & 22 & 154 & 84 \\
\hline Gross official reserves $6 /$ & 475 & 489 & 492 & 514 & 667 & 751 \\
\hline In months of next year's imports & 2.4 & 2.3 & 2.1 & 1.9 & 2.1 & 2.7 \\
\hline \multicolumn{7}{|l|}{ Memorandum items: } \\
\hline \multicolumn{7}{|l|}{ Exchange rates } \\
\hline Brunei dollars per U.S. dollar (period average) & 1.74 & 1.69 & 1.66 & 1.59 & 1.51 & 1.41 \\
\hline Brunei dollars per U.S. dollar (end of period) & 1.70 & 1.63 & 1.66 & 1.54 & 1.44 & 1.44 \\
\hline Nominal effective exchange rates $(2000=100)$ & 97.0 & 96.4 & 97.2 & 100.0 & 101.1 & 105.7 \\
\hline Real effective exchange rates $(2000=100)$ & 93.2 & 90.4 & 89.3 & 89.8 & 88.8 & 90.5 \\
\hline Nominal GDP (in millions of U.S. dollars) & 6,557 & 7,872 & 9,531 & 11,470 & 12,248 & 14,417 \\
\hline
\end{tabular}

Sources: Data provided by the Brunei authorities; and Fund staff estimates.

1/ Balance of payments data for 2003 onwards were revised after the 2004 Article IV consultation.

2/ From 2004, excludes re-exports.

3/ Based on Fund staff estimates.

4/ Includes changes in banks' net foreign assets.

5/ The large scale of errors and omissions reflects the lack of comprehensive balance of payments data.

6/ Includes Brunei Currency and Monetary Board's foreign exchange assets, SDR holdings and reserve position in the Fund. 
Table 31. Brunei Darussalam: Exports by Commodity, 2002-09

\begin{tabular}{|c|c|c|c|c|c|c|c|c|}
\hline & 2002 & 2003 & 2004 & 2005 & 2006 & 2007 & 2008 & $\begin{array}{r}2009 \\
\mathrm{H} 1\end{array}$ \\
\hline & \multicolumn{8}{|c|}{ (In millions of U.S. dollars) } \\
\hline Total exports & 3,702 & 4,421 & 5,057 & 6,249 & 7,608 & 7,668 & 10,421 & 3,525 \\
\hline Food and live animals & 1 & 2 & 2 & 4 & 6 & 5 & 3 & 1 \\
\hline Beverages and tobacco & 0 & 0 & 0 & 0 & 0 & 0 & 0 & 0 \\
\hline Crude material inedible & 2 & 3 & 6 & 7 & 9 & 7 & 9 & 1 \\
\hline Mineral fuels & 3,259 & 3,876 & 4,624 & 5,886 & 7,328 & 7,373 & 10,193 & 3,384 \\
\hline Animal and vegetable oils and fats & 0 & 0 & 0 & 0 & 0 & 0 & 0 & 0 \\
\hline Chemicals & 1 & 3 & 2 & 3 & 3 & 3 & 4 & 3 \\
\hline Manufactured goods & 40 & 54 & 24 & 24 & 19 & 25 & 28 & 9 \\
\hline Machinery and transport equipment $1 /$ & 171 & 236 & 140 & 125 & 94 & 108 & 80 & 87 \\
\hline Miscellaneous manufactured articles & 222 & 240 & 250 & 191 & 138 & 138 & 98 & 38 \\
\hline \multirow{2}{*}{ Other } & 6 & 7 & 9 & 9 & 12 & 9 & 6 & 3 \\
\hline & \multicolumn{8}{|c|}{ (In percent of total exports) } \\
\hline Total exports & 100.0 & 100.0 & 100.0 & 100.0 & 100.0 & 100.0 & 100.0 & 100.0 \\
\hline Food and live animals & 0.0 & 0.0 & 0.0 & 0.1 & 0.1 & 0.1 & 0.0 & 0.0 \\
\hline Beverages and tobacco & 0.0 & 0.0 & 0.0 & 0.0 & 0.0 & 0.0 & 0.0 & 0.0 \\
\hline Crude material inedible & 0.1 & 0.1 & 0.1 & 0.1 & 0.1 & 0.1 & 0.1 & 0.0 \\
\hline Mineral fuels & 88.0 & 87.7 & 91.4 & 94.2 & 96.3 & 96.2 & 97.8 & 96.0 \\
\hline Animal and vegetable oils and fats & 0.0 & 0.0 & 0.0 & 0.0 & 0.0 & 0.0 & 0.0 & 0.0 \\
\hline Chemicals & 0.0 & 0.1 & 0.0 & 0.0 & 0.0 & 0.0 & 0.0 & 0.1 \\
\hline Manufactured goods & 1.1 & 1.2 & 0.5 & 0.4 & 0.2 & 0.3 & 0.3 & 0.3 \\
\hline Machinery and transport equipment & 4.6 & 5.3 & 2.8 & 2.0 & 1.2 & 1.4 & 0.8 & 2.5 \\
\hline Miscellaneous manufactured articles & 6.0 & 5.4 & 4.9 & 3.1 & 1.8 & 1.8 & 0.9 & 1.1 \\
\hline Other & 0.2 & 0.2 & 0.2 & 0.1 & 0.2 & 0.1 & 0.1 & 0.1 \\
\hline
\end{tabular}

Source: Brunei Darussalam Statistical Yearbook, Department of Economic Planning and Development.

1/ Excludes re-exports from 2004. 
Table 32. Brunei Darussalam: Value and Destination of Selected Exports, 2002-09

$\begin{array}{llllllll}2002 & 2003 & 2004 & 2005 & 2006 & 2007 & 2008 & 2009\end{array}$

$\mathrm{H} 1$

(In millions of Brunei dollars)

Value of major exports

Petroleum

Crude petroleum

Petroleum products

Natural gas

$\begin{array}{rrrrrrrr}3,232 & 3,777 & 4,913 & 6,536 & 8,158 & 7,627 & 7,949 & 1,984 \\ 3,140 & 3,748 & 4,913 & 6,536 & 8,158 & 7,627 & 7,949 & 1,984 \\ 92 & 29 & \ldots & \ldots & \ldots & \ldots & \ldots & \ldots \\ 2,603 & 2,965 & 2,914 & 3,258 & 3,514 & 3,484 & 6,667 & 3,069\end{array}$

(In millions of U.S. dollars)

Petroleum
Crude petroleum
Petroleum products
Natural gas

Major exports by destination

Crude petroleum
Japan
Korea, Republic of
Philippines
Singapore
Thailand
United States
Petroleum products
Japan
Korea, Republic of
Singapore
United States
Natural gas
Japan
Korea, Republic of

\begin{tabular}{rrrrrrrr}
1,805 & 2,174 & 2,902 & 3,928 & 5,122 & 5,061 & 5,544 & 1,329 \\
1,754 & 2,157 & 2,902 & 3,928 & 5,122 & 5,061 & 5,544 & 1,329 \\
51 & 17 & $\ldots$ & $\ldots$ & $\ldots$ & $\ldots$ & $\ldots$ & $\ldots$ \\
1,454 & 1,702 & 1,722 & 1,958 & 2,206 & 2,312 & 4,650 & 2,055 \\
\multicolumn{1}{c}{ (In millions of Brunei dollars) }
\end{tabular}

$\begin{array}{rrrrrrrr}3,140 & 3,748 & 4,913 & 6,536 & 8,158 & 7,627 & 7,949 & 1,984 \\ 376 & 467 & 664 & 832 & 598 & 649 & 479 & 35 \\ 442 & 586 & 750 & 1,040 & 1,420 & 1,133 & 1,519 & 392 \\ 40 & \ldots & \ldots & \ldots & \ldots & \ldots & 214 & \ldots \\ 57 & \ldots & \ldots & 31 & 151 & 34 & 107 & 41 \\ 877 & 724 & 589 & 287 & 227 & 154 & 113 & 132 \\ 186 & 287 & 293 & 534 & 655 & 430 & \ldots & \ldots\end{array}$

$92 \quad 29$

$23 \quad \ldots$

$38 \quad 16$

... 14

31

$2,572 \quad 2,943$

$2,274 \quad 2,684 \quad 2,564$

$297 \quad 259$
$\ldots$
$\cdots$
$\cdots$

2,983

275

$\begin{array}{rrrr}3,514 & 3,484 & 6,667 & 3,069 \\ 3,109 & 3,215 & 5,975 & 2,892 \\ 405 & 269 & 692 & 177\end{array}$

Source: Brunei Darussalam Statistical Yearbook, Department of Economic Planning and Development. 
Table 33. Brunei Darussalam: Exports by Country of Destination, 2002-09

(In millions of U.S. dollars)

\begin{tabular}{|c|c|c|c|c|c|c|c|c|}
\hline & 2002 & 2003 & 2004 & 2005 & 2006 & 2007 & 2008 & $\begin{array}{r}2009 \\
\mathrm{H} 1\end{array}$ \\
\hline ASEAN & 831 & 892 & 868 & 1,532 & 1,885 & 2,153 & 2,517 & 504 \\
\hline \multicolumn{9}{|l|}{ Of which: } \\
\hline Indonesia & 31 & 113 & 316 & 1,163 & 1,510 & 1,853 & 2,083 & 289 \\
\hline Malaysia & 134 & 163 & 70 & 58 & 40 & 61 & 30 & 61 \\
\hline Philippines & 23 & 1 & 1 & 2 & 1 & 1 & 150 & 1 \\
\hline Singapore & 152 & 198 & 132 & 135 & 190 & 133 & 174 & 64 \\
\hline Thailand & 491 & 417 & 349 & 174 & 144 & 106 & 80 & 88 \\
\hline European Union & 22 & 15 & 31 & 20 & 18 & 18 & 14 & 3 \\
\hline \multicolumn{9}{|l|}{ Of which: } \\
\hline France & 1 & 0 & 1 & 1 & 1 & 1 & 1 & 0 \\
\hline Germany & 3 & 4 & 4 & 3 & 4 & 5 & 2 & 1 \\
\hline United Kingdom & 7 & 9 & 21 & 13 & 10 & 9 & 11 & 2 \\
\hline Others & 2,849 & 3,514 & 4,158 & 4,697 & 5,705 & 5,497 & 7,885 & 3,017 \\
\hline \multicolumn{9}{|l|}{ Of which: } \\
\hline Australia & 326 & 370 & 593 & 603 & 930 & 1,051 & 1,080 & 179 \\
\hline Japan & 1,494 & 1,811 & 1,908 & 2,293 & 2,329 & 2,565 & 4,502 & 1,961 \\
\hline Korea, Republic of & 434 & 494 & 650 & 790 & 1,146 & 930 & 1,542 & 381 \\
\hline Taiwan Province of China & 0 & 0 & 4 & 1 & 0 & 1 & 1 & 1 \\
\hline United States & 292 & 343 & 349 & 466 & 512 & 386 & 66 & 31 \\
\hline Total & 3,702 & 4,421 & 5,057 & 6,249 & 7,608 & 7,668 & 10,421 & 3,525 \\
\hline
\end{tabular}

Source: Brunei Darussalam Statistical Yearbook, Department of Economic Planning and Development. 
Table 34. Brunei Darussalam: Imports by Commodity, 2002-09

\begin{tabular}{|c|c|c|c|c|c|c|c|c|}
\hline & 2002 & 2003 & 2004 & 2005 & 2006 & 2007 & 2008 & 2009 \\
\hline & \multicolumn{8}{|c|}{ (In millions of U.S. dollars) } \\
\hline Total imports (c.i.f.) & 1,556 & 1,327 & 1,422 & 1,491 & 1,669 & 2,101 & 1,137 & 1,260 \\
\hline Food and live animals & 179 & 200 & 210 & 259 & 235 & 270 & 156 & 157 \\
\hline Beverages and tobacco & 24 & 25 & 38 & 41 & 41 & 51 & 27 & 25 \\
\hline Crude material inedible & 13 & 14 & 12 & 9 & 14 & 14 & 7 & 10 \\
\hline Mineral fuels & 3 & 11 & 11 & 25 & 28 & 45 & 21 & 27 \\
\hline Animal and vegetable oils and fats & 6 & 9 & 7 & 6 & 7 & 9 & 7 & 4 \\
\hline Chemicals & 95 & 101 & 113 & 138 & 175 & 157 & 93 & 90 \\
\hline Manufactured goods & 372 & 341 & 354 & 367 & 401 & 457 & 283 & 287 \\
\hline Machinery and transport equipments & 714 & 468 & 497 & 478 & 588 & 870 & 438 & 520 \\
\hline Miscellaneous manufactured articles & 146 & 152 & 174 & 161 & 173 & 216 & 100 & 135 \\
\hline \multirow[t]{2}{*}{ Other } & 4 & 6 & 6 & 7 & 8 & 11 & 5 & 6 \\
\hline & \multicolumn{8}{|c|}{ (In percent of total imports) } \\
\hline Total imports (c.i.f.) & 100.0 & 100.0 & 100.0 & 100.0 & 100.0 & 100.0 & 100.0 & 100.0 \\
\hline Food and live animals & 11.5 & 15.1 & 14.8 & 17.4 & 14.1 & 12.9 & 13.7 & 12.4 \\
\hline Beverages and tobacco & 1.5 & 1.9 & 2.7 & 2.7 & 2.5 & 2.4 & 2.4 & 2.0 \\
\hline Crude material inedible & 0.8 & 1.1 & 0.8 & 0.6 & 0.9 & 0.7 & 0.6 & 0.8 \\
\hline Mineral fuels & 0.2 & 0.8 & 0.8 & 1.7 & 1.7 & 2.1 & 1.9 & 2.1 \\
\hline Animal and vegetable oils and fats & 0.4 & 0.7 & 0.5 & 0.4 & 0.4 & 0.4 & 0.6 & 0.3 \\
\hline Chemicals & 6.1 & 7.6 & 7.9 & 9.3 & 10.5 & 7.5 & 8.2 & 7.1 \\
\hline Manufactured goods & 23.9 & 25.7 & 24.9 & 24.6 & 24.0 & 21.8 & 24.9 & 22.8 \\
\hline Machinery and transport equipments & 45.9 & 35.3 & 35.0 & 32.1 & 35.2 & 41.4 & 38.5 & 41.3 \\
\hline Miscellaneous manufactured articles & 9.4 & 11.5 & 12.2 & 10.8 & 10.4 & 10.3 & 8.8 & 10.7 \\
\hline Other & 0.3 & 0.5 & 0.4 & 0.5 & 0.4 & 0.5 & 0.4 & 0.5 \\
\hline
\end{tabular}

Source: Brunei Darussalam Statistical Yearbook, Department of Economic Planning and Development. 
Table 35. Brunei Darussalam: Imports by Country of Origin, 2002-09

(In millions of U.S. dollars)

\begin{tabular}{|c|c|c|c|c|c|c|c|c|}
\hline & 2002 & 2003 & 2004 & 2005 & 2006 & 2007 & 2008 & $\begin{array}{r}2009 \\
\mathrm{H} 1\end{array}$ \\
\hline $\begin{array}{l}\text { ASEAN } \\
\text { Of which: }\end{array}$ & 613 & 629 & 650 & 759 & 796 & 1,041 & 1,218 & 704 \\
\hline Indonesia & 38 & 36 & 31 & 34 & 47 & 62 & 68 & 29 \\
\hline Malaysia & 233 & 269 & 287 & 364 & 361 & 398 & 475 & 239 \\
\hline Philippines & 3 & 3 & 4 & 6 & 6 & 7 & 17 & 3 \\
\hline Singapore & 282 & 267 & 262 & 279 & 290 & 472 & 520 & 374 \\
\hline Thailand & 57 & 54 & 66 & 76 & 92 & 104 & 133 & 57 \\
\hline $\begin{array}{l}\text { European Union } \\
\text { Of which: }\end{array}$ & 198 & 143 & 172 & 142 & 172 & 231 & 264 & 127 \\
\hline France & 17 & 22 & 14 & 11 & 17 & 23 & 25 & 25 \\
\hline Germany & 45 & 43 & 61 & 33 & 40 & 58 & 75 & 27 \\
\hline Italy & 12 & 9 & 11 & 11 & 36 & 17 & 33 & 17 \\
\hline Netherlands & 8 & 6 & 13 & 11 & 15 & 22 & 22 & 9 \\
\hline United Kingdom & 99 & 49 & 53 & 58 & 46 & 78 & 65 & 33 \\
\hline $\begin{array}{l}\text { Others } \\
\text { Of which: }\end{array}$ & 745 & 555 & 600 & 590 & 701 & 829 & 1,025 & 416 \\
\hline Australia & 41 & 40 & 40 & 33 & 33 & 35 & 36 & 17 \\
\hline China & 60 & 65 & 88 & 94 & 131 & 158 & 176 & 64 \\
\hline Hong Kong SAR & 64 & 87 & 73 & 50 & 55 & 52 & 66 & 12 \\
\hline Japan & 214 & 133 & 140 & 136 & 213 & 178 & 216 & 118 \\
\hline Korea, Republic of & 31 & 31 & 40 & 61 & 28 & 34 & 82 & 30 \\
\hline Taiwan Province of China & 20 & 20 & 21 & 26 & 28 & 43 & 45 & 18 \\
\hline United States & 288 & 153 & 167 & 145 & 151 & 268 & 349 & 129 \\
\hline Total (c.i.f.) & 1,556 & 1,327 & 1,422 & 1,491 & 1,669 & 2,101 & 2,543 & 1,260 \\
\hline
\end{tabular}

Source: Brunei Darussalam Statistical Yearbook, Department of Economic Planning and Development. 
Table 36. Brunei Darussalam: Exchange Rates, 2004-09 1/

\begin{tabular}{|c|c|c|c|c|c|c|c|c|c|c|c|c|c|c|c|c|c|c|c|c|c|c|c|c|}
\hline & \multicolumn{4}{|c|}{2004} & \multicolumn{4}{|c|}{2005} & \multicolumn{4}{|c|}{2006} & \multicolumn{4}{|c|}{2007} & \multicolumn{4}{|c|}{2008} & \multicolumn{4}{|c|}{2009} \\
\hline & Q1 & Q2 & Q3 & $\overline{\mathrm{Q} 4}$ & Q1 & Q2 & Q3 & $\overline{\mathrm{Q4}}$ & Q1 & Q2 & Q3 & Q4 & Q1 & Q2 & Q3 & $\overline{\mathrm{Q} 4}$ & Q1 & Q2 & Q3 & $\overline{\mathrm{Q} 4}$ & Q1 & $\bar{Q} 2$ & Q3 & $\overline{Q 4}$ \\
\hline \multicolumn{25}{|c|}{ Brunei dollar per U.S. dollar } \\
\hline Period average & 1.69 & 1.70 & 1.71 & 1.66 & 1.64 & 1.66 & 1.68 & 1.69 & 1.63 & 1.59 & 1.58 & 1.56 & 1.53 & 1.52 & 1.52 & 1.45 & 1.41 & 1.37 & 1.40 & 1.49 & 1.51 & 1.47 & 1.44 & 1.39 \\
\hline End of period & 1.68 & 1.72 & 1.69 & 1.63 & 1.65 & 1.68 & 1.69 & 1.66 & 1.62 & 1.59 & 1.59 & 1.53 & 1.52 & 1.53 & 1.49 & 1.44 & 1.38 & 1.36 & 1.43 & 1.44 & 1.52 & 1.45 & 1.41 & 1.4 \\
\hline \multicolumn{25}{|c|}{ Brunei dollar per SDR } \\
\hline End of period & 2.49 & 2.52 & 2.48 & 2.54 & 2.49 & 2.45 & 2.45 & 2.38 & 2.33 & 2.35 & 2.34 & 2.31 & 2.29 & 2.32 & 2.32 & 2.28 & 2.27 & 2.22 & 2.23 & 2.22 & 2.27 & 2.25 & 2.24 & 2.20 \\
\hline \multicolumn{25}{|c|}{ Yen per Brunei dollar } \\
\hline Period average & 63.24 & 64.39 & 64.37 & 63.86 & 63.85 & 64.82 & 66.37 & 69.46 & 71.74 & 71.98 & 73.57 & 75.51 & 77.95 & 79.17 & 77.56 & 77.69 & 74.65 & 76.50 & 76.98 & 64.54 & 61.81 & 66.02 & 65.00 & 64.36 \\
\hline End of period & 62.29 & 63.26 & 65.58 & 62.83 & 64.98 & 65.50 & 66.90 & 70.48 & 72.55 & 72.37 & 74.24 & 77.60 & 77.64 & 80.51 & 77.30 & 77.69 & 72.39 & 78.01 & 72.82 & 62.80 & 64.72 & 66.16 & 63.48 & 65.82 \\
\hline \multicolumn{25}{|c|}{ Brunei dollar per euro } \\
\hline Period average & 2.12 & 2.05 & 2.09 & 2.15 & 2.15 & 2.09 & 2.04 & 2.01 & 1.96 & 2.00 & 2.01 & 2.01 & 2.01 & 2.06 & 2.08 & 2.11 & 2.11 & 2.13 & 2.10 & 1.96 & 1.97 & 2.01 & 2.06 & 2.06 \\
\hline End of period & 2.06 & 2.07 & 2.08 & 2.22 & 2.13 & 2.04 & 2.04 & 1.98 & 1.97 & 2.02 & 2.02 & 2.02 & 2.02 & 2.06 & 2.11 & 2.13 & 2.18 & 2.15 & 2.06 & 2.03 & 2.02 & 2.05 & 2.07 & 2.02 \\
\hline
\end{tabular}

Sources: IMF, International Financial Statistics; and Monetary Authority of Singapore websites.

1/ Exchange rates for the Brunei dollar are based on Singapore dollar rates reported to IMF, International Financial Statistics. Under a currency board arrangement, the Brunei dollar is maintained at par with the Singapore dollar. 